\title{
Very viscous horizontal convection
}

\author{
S. CHIU - WEBSTER, E. J. HINCH AND J. R. LISTER \\ Department of Applied Mathematics and Theoretical Physics, University of Cambridge, \\ Wilberforce Rd, Cambridge CB3 0WA, UK
}

(Received 30 May 2007 and in revised form 28 May 2008)

'Horizontal convection' arises when a temperature variation is imposed along a horizontal boundary of a finite fluid volume. Here we study the infinite-Prandtlnumber limit relevant to very viscous fluids, motivated by the study of convection in glass furnaces. We consider a rectangular domain with insulating conditions on the sides and bottom, and a linear temperature gradient on the top. We describe steady states for a large range of aspect ratio $A$ and Rayleigh number $R a$, and find universal scalings for the transition from small to large Rayleigh numbers. At large Rayleigh number, the top boundary-layer thickness scales as $R a^{-1 / 5}$, with the circulation and heat flux scaling as $R a^{1 / 5}$. These scalings hold for both rigid and shear-free boundary conditions on the top or on the other boundaries, which is initially surprising, but is because the return flow is dominated by a horizontal intrusion immediately beneath the top boundary layer. A downwelling plume also forms on one side, but because of strong stratification in the interior, the volume flux it carries is much smaller than that of the horizontal intrusion, decaying as the inverse of the depth below the top boundary. The fluid in the plume detrains into the interior and then returns to the top boundary, thus forming a 'filling box'. We find analytic solutions for the interior temperature and streamfunction and match them to a similarity solution for the plume. At depths comparable to the length of the top boundary the streamfunction has $O(1)$ values and the temperature variations scale as $1 / R a$. Transient calculations with a large, but finite, Prandtl number, show how the steady state is reached from hot and cold initial conditions.

\section{Introduction}

The temperature of the upper surface of the molten glass in glass furnaces varies from one end to the other due to the addition of cold floating raw materials at one end and heating by gas flames from above. The resulting convection in the furnace is an example of horizontal convection, which is the general term for convection driven by varying the temperature along a horizontal surface. The glass furnace motivates an investigation of horizontal convection in the very viscous limit of infinite Prandtl number, and poses a simple theoretical problem in the study of convective motions of fluids, in the same canonical vein as the problems of Bénard and vertical-slot convection. Horizontal convection differs in several ways from these systems. In particular, as we shall show, at large Rayleigh number only a weak heat flux and flow penetrate the depth of the fluid.

Previous studies of horizontal convection have been motivated by the consideration of transport of warm fluid in the oceans from the tropics to high latitudes, where it cools and sinks, before an upwelling flow across the ocean basin completes the flow (see Huang 1999; Wunsch \& Ferrari 2004; Hughes \& Griffiths 2006, and 
references therein). The sinking regions and upwelling are important for establishing the stratification of the ocean.

A number of authors have considered idealized horizontal convection, using laboratory experiments, two-dimensional numerical simulations, and theoretical analyses valid for Prandtl number $\operatorname{Pr} \gtrsim O(1)$. Two of the questions that recur in the literature are the correct scaling dependences at large Rayleigh number, and the depth and strength of the return flow. We shall resolve these issues for the very viscous limit, giving a detailed analysis supported by numerical simulation.

In a foundational paper, Rossby (1965) described laboratory experiments for Prandtl numbers between 10 and $10^{4}$ and Rayleigh numbers between $10^{7}$ and $10^{10}$, and presented a scaling argument that predicts that both the circulation and heat flux scale as $R a^{1 / 5}$. These scalings are often referred to as the Rossby scalings. Rossby (1998) presented numerical simulations with shear-free boundary conditions, Rayleigh numbers up to $10^{8}$, and Prandtl numbers between 1 and 100. It was suggested, without detailed justification, that the same $R a^{1 / 5}$ scalings remain valid despite the change to shear-free boundary conditions. There was good numerical evidence that the heat flux scales as $R a^{1 / 5}$, but the evidence that the streamfunction scales as $R a^{1 / 5}$ was less clear.

Further laboratory experiments were conducted, for a rigid top and $P r \simeq 8$, by Wang \& Huang (2005) and Mullarney, Griffiths \& Hughes (2004), with Rayleigh numbers in the ranges $10^{7}-10^{10}$ and $10^{11}-10^{13}$ respectively. Wang \& Huang (2005) found a transition to unsteady behaviour at $R a=4 \times 10^{8}$, whereas Mullarney et al. (2004) observed that the flow was always unsteady for their very large Rayleigh numbers and apparently turbulent. Another difference between the two sets of experiments was the 'partial penetration' observed by Wang \& Huang (2005), with most of the return flow restricted to a shallow layer near the heated boundary, which contrasts with the more significant interior flow found by Mullarney et al. (2004) using larger Rayleigh numbers. Both groups presented good evidence for the $R a^{1 / 5}$ scalings in the unsteady regime, and Mullarney et al. (2004) showed that the $R a^{1 / 5}$ scalings were consistent with four numerical experiments conducted at Rayleigh numbers around $10^{12}$. Paparella \& Young (2002) described numerical simulations with stressfree conditions and Prandtl numbers between 0.1 and 10 , and found the stability boundary in the parameter space $(R a, P r)$ for $P r \leqslant 4$.

Theoretical analyses, valid for $\operatorname{Pr} \gtrsim O(1)$, have also been undertaken. Killworth \& Manins (1980) conducted a boundary-layer analysis, and derived a similarity solution for the main boundary layer by imposing a quadratic temperature profile. Hughes et al. (2007) studied a filling-box model of horizontal convection at large Rayleigh number. In their model the return flow comprises a turbulent plume, which evolves by buoyancy and entrainment near a surface heat sink, and solely by entrainment further below the surface. The plume reaches the bottom of the tank, and then spreads across the width before returning as a uniform upwelling through the interior.

The question of the correct asymptotic scaling has also been taken up by Siggers, Kerswell \& Balmforth (2004), who derived a bound for the heat flux of $R a^{1 / 3}$ and also presented numerical results for Rayleigh numbers up to $10^{8}$ and Prandtl numbers between 0.2 and 4, with stress-free conditions. Daniels \& Punpocha $(2004,2005)$ presented a theoretical analysis of horizontal convection in a porous medium, which gives different scalings, but is in much the same spirit as the analysis herein. For infinite-Prandtl-number horizontal convection, some numerical results were described in McKenzie, Roberts \& Weiss (1974). 
Recently, Gramberg, Howell \& Ockendon (2007) looked at the problem of very viscous horizontal convection addressed in this paper, but with different conclusions. For large Rayleigh numbers, they constructed an asymptotic theory treating the hot boundary layer like the spreading of a thin layer of light liquid over a shallow layer of heavy fluid, as in Lister \& Kerr (1989). The condition of no tangential stress on the upper free surface and the assumption that the return flow is distributed over the depth of the shallow layer makes the thin light layer move at a uniform velocity to the leading approximation. Considering additionally the thermal balance in the thin top boundary layer leads to a prediction that the thickness of the thin layer scales with $R a^{-1 / 4}$. Gramberg et al. (2007) did not test this prediction or associated predictions against their numerical solutions, conducted for Rayleigh numbers up to $7 \times 10^{8}$. We do not find this behaviour because the return flow is not actually distributed over the depth of the layer for reasons that are explained in $\S 4$. In fact, the velocity profiles in figures 11, 12 and 13 of Gramberg et al. (2007) do not show the uniform velocity across the top layer which is the basis of their analysis.

In this paper we largely neglect inertia and consider the infinite-Prandtl-number limit of horizontal convection, as appropriate for the very viscous fluids found in a glass furnace and the Earth's mantle. We focus on steady states, and briefly consider evolution toward steady state. We are able to examine and explain the structure of the steady solutions at large Rayleigh number, and derive the key scalings for the different regions. The theoretical analysis is guided and supported by numerical simulations for Rayleigh numbers up to $10^{10}$. We present conclusive numerical evidence that, for both rigid and shear-free conditions, at large Rayleigh number the boundary-layer thickness scales as $R a^{-1 / 5}$, with circulation strength and heat flux scaling as $R a^{1 / 5}$.

The independence of the scalings of the boundary condition is at first surprising, since standard assumptions about viscous boundary layers typically yield different answers depending on whether the boundaries are rigid or stress-free (e.g. Roberts 1977; Huppert 1982; Lister \& Kerr 1989). The results are understood by examining the return flow from the top boundary layer, and showing that it is dominated by a horizontal intrusion immediately below the boundary layer, with only a weak downwelling plume that returns through the interior at depth. The presence of the intrusion implies strong shear within the boundary layer even for a shear-free surface condition. It is therefore valid to assume that the characteristic vertical length scale of velocity gradients is the same as that of the temperature gradients, which gives rise to the $\mathrm{Ra}^{1 / 5}$ scalings. We also show that the streamfunction decays as the inverse of the depth for large depths, because of strong stratification beneath the top boundary layer. The streamfunction at depth is $O(1)$ with temperature variations scaling as $1 / R a$. We find analytic solutions for the interior, and match them to a similarity solution of the lubrication and thermal boundary-layer equations for the plume. The predictions are in excellent agreement with numerical simulations.

We also study the effect of aspect ratio $A$ (height over width) and the transition from small to large Rayleigh numbers, and find universal scalings for the circulation strength and heat flux. The parameter governing the transition is $A^{5} R a$ if $A \ll 1$ and $R a$ if $A>1$, where $R a$ is as defined in (2.3).

The paper is organized as follows. In $\S 2$ we present the non-dimensional governing equations and explain the numerical method used to solve them. In $\S 3$ we describe the transition from small to large Rayleigh numbers, and derive universal scalings that collapse the results for different aspect ratios. In $\S 4$ we study the structure of the large- $R a$ regime. We first explain how the return flow comprises a horizontal intrusion and a side plume, and transients of numerical simulations are described to support 


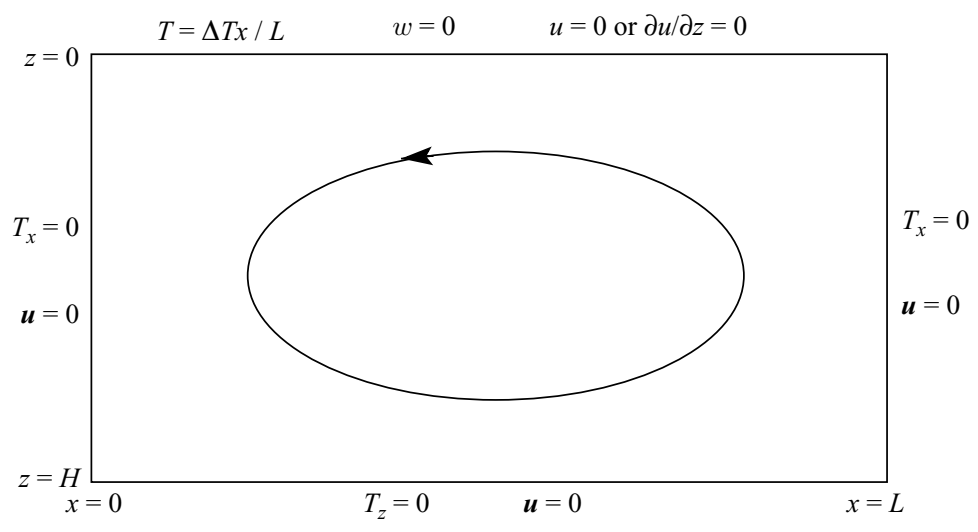

FIGURE 1. Geometry and boundary conditions of the idealized dimensional problem.

these ideas. We then examine the different regions of the flow: the top boundary layer, the intrusion, the side plume, and the return flow through the interior. In $\S 5$ we discuss our results.

\section{Governing equations and numerical method}

Consider a two-dimensional rectangular box of length $L$ and height $H$, and define coordinates $(x, z)$ with the origin at the top left corner and $z$ taken as positive downwards (figure 1). Flow is driven by a linear temperature profile $T=\Delta T x / L$ imposed along the top boundary $z=0$; all other walls are insulating so that the effect of horizontal convection can be considered in isolation. (Mild singularities in the corners due to the jump in $\mathrm{d} T / \mathrm{d} x$ do not have a significant effect on the flow.) One advantage of a linear temperature profile is that it is easy to realize experimentally. A different temperature profile is expected to give similar results, provided it is monotonic and smooth.

We consider rigid and shear-free conditions for the top boundary, and concentrate on a rigid condition for the sides and bottom. As discussed in $\S 5$, the results for shear-free side and bottom boundaries are very similar. We adopt the Boussinesq approximation, and, for simplicity, the material properties of kinematic viscosity $v$, thermal diffusivity $\kappa$ and thermal expansivity $\alpha$ are assumed to be constant. We consider the limit of infinite Prandtl number, so that fluid inertia is neglected.

We non-dimensionalize both $x$ and $z$ with the box length $L$, rather than the height $H$, because $L$ is more appropriate for the boundary-layer scalings in $\S 3.2$. The streamfunction $\psi$ is non-dimensionalized with $\kappa$ (so that the velocity $\boldsymbol{u}$ is non-dimensionalized with $\kappa / L$ ) and the temperature $T$ with $\Delta T$. Then the steady non-dimensional governing equations for vorticity and heat conservation are

$$
\begin{gathered}
\nabla^{4} \psi=R a \frac{\partial T}{\partial x}, \\
-\frac{\partial \psi}{\partial z} \frac{\partial T}{\partial x}+\frac{\partial \psi}{\partial x} \frac{\partial T}{\partial z}=\nabla^{2} T
\end{gathered}
$$

where

$$
R a=\frac{g \alpha \Delta T L^{3}}{\nu \kappa} .
$$


The only dimensionless parameters are the Rayleigh number $R a$, and the aspect ratio

$$
A=H / L \text {. }
$$

Of particular interest are global measures of the strength of the flow and the heat transfer in steady state. For the strength of the flow, we use the Péclet number defined by

$$
P e \equiv \psi_{\max } .
$$

For the global heat transfer, we use the unsigned Nusselt number

$$
N u \equiv \int_{0}^{1}\left|\frac{\partial T}{\partial z}(z=0)\right| \mathrm{d} x
$$

because the net heat transfer through the top boundary is zero in steady state, as the other boundaries are insulating.

\subsection{Numerical method}

To find solutions of (2.1) and (2.2) numerically, we evolved the equations

$$
\begin{gathered}
\frac{\partial \boldsymbol{u}}{\partial t}=-\nabla p+\nabla^{2} \boldsymbol{u}-R a T \hat{\boldsymbol{z}}, \\
\nabla \cdot \boldsymbol{u}=0, \\
\frac{\partial T}{\partial t}+\nabla \cdot(\boldsymbol{u} T)=\nabla^{2} T
\end{gathered}
$$

for the primitive variables $\boldsymbol{u}, p$ and $T$ until they reached a steady state. Equation (2.7) is a form of artificial time-evolution, which reduces to (2.1) in steady state. The numerical evolution scheme employs a finite-volume method with a staggered grid, and it conserves mass, momentum and heat exactly. With Crank-Nicolson timestepping, the method is $O\left(\Delta x^{2}\right)$ in space and $O\left(\Delta t^{2}\right)$ in time. The pressure is obtained at each integration step with a pressure update method. The Poisson problems for the pressure, velocity and temperature are solved with a multigrid method.

At each time step, the greatest numerical cost results from the Poisson solver, which is $O\left(N^{2}\right)$ per time step using a multigrid method, where $N$ is the number of grid points in each direction. At each time-step the multigrid solver was iterated either once or twice. An $O(1)$ time in (2.7)-(2.9) was required to reach steady state, and hence the required number of time-steps $\sim 1 / \Delta t \sim 1 / \Delta x \sim N$, where the link between $\Delta t$ and $\Delta x$ is a Courant condition. Combining the cost of the multigrid solver and the required number of iterations, the total cost of the numerical algorithm was $O\left(N^{3}\right)$. The dependence of the cost on Rayleigh number is asymptotically $O\left(R a^{7 / 15}\right)$, which as we shall see follows from the Courant condition for the vertical flow in the corner region.

In order to resolve the thin boundary layers at large Rayleigh number, we used a smoothly varying coordinate transformation that stretches the grid in the vicinity of the boundaries without losing the accuracy of central-differencing. Since the numerical errors in a boundary layer of width $\delta$ are $O\left(\Delta x^{2} / \delta^{2}\right)$ and in the interior are $O\left(\Delta x^{2}\right)$, it is a considerable advantage to use a stretched grid that makes these errors similar so that the boundary layer is not under-resolved or the interior over-resolved. For $R a=10^{8}$ and $A=1$, for example, a stretched grid required approximately five times fewer points to achieve the same degree of accuracy as a uniform grid. This reduction in $N$ by a factor 5 decreased the computational cost by a factor of about 100 . For still larger values of $R a$, and therefore thinner boundary layers, the use of a stretched grid 


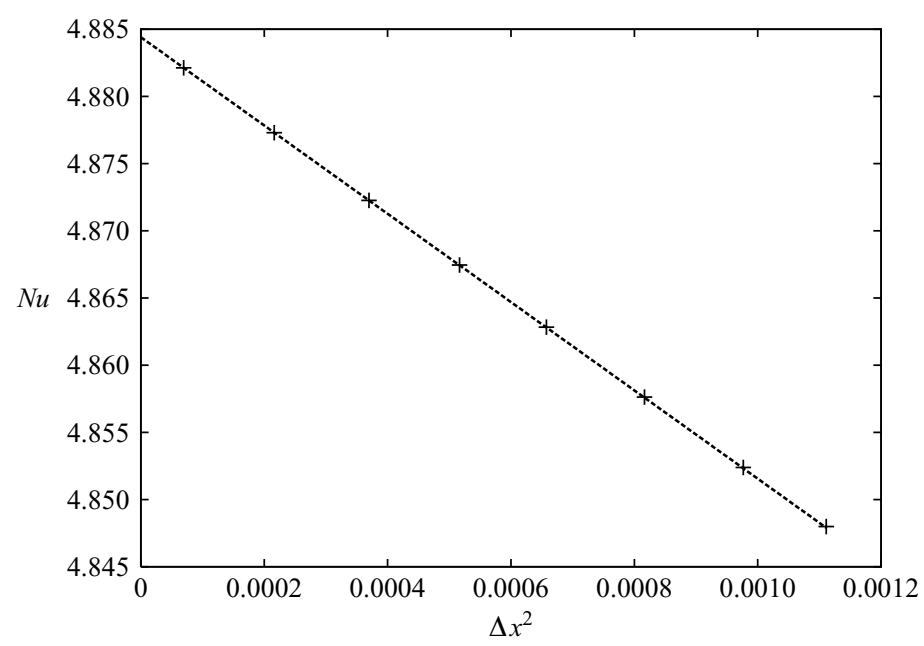

FIGURE 2. Example of $\Delta x^{2}$ convergence and Richardson extrapolation. Nusselt number $N u$ against different grid spacings $\Delta x$ for Bénard convection at $R a=10^{4}$ and $A=1$. The dashed line extrapolates the two data points with the smallest values of $\Delta x^{2}$, giving the value 4.884 as $\Delta x^{2} \rightarrow 0$.

is even more important to achieve practical inexpensive results. We used stretched grids of size up to $200 \times 200$, in which the smallest and largest grid spacings differed by a factor of up to 20 in the horizontal direction, and up to 40 in the vertical direction.

Benchmark tests were carried out to validate the numerical code, and the results compared to the literature. Figure 2 shows the Nusselt number computed at different spatial resolutions for steady-state Bénard convection at $R a=10^{4}$ and $A=1$ (with $\operatorname{Pr}=\infty$ ), and all walls rigid. This figure, and similar results, confirms the code to be $O\left(\Delta x^{2}\right)$. Richardson extrapolation of the two data points with the smallest values of $\Delta x^{2}$ gives a grid-independent value $N u=4.884$. This Nusselt number, and similarly obtained root-mean-square velocity and corner heat fluxes, all agreed with the benchmark results reported in Blankenbach et al. (1989) to at least four significant figures.

In the numerical results reported below for horizontal convection, second-order convergence was again verified, and Richardson extrapolation was used on the finest grids to obtain highly accurate numerical data for all the scalar quantities. Following this procedure usually ensured accuracy to at least three significant figures, so that error bars are insignificant on the scale of the graphs presented.

We note that for the range of Rayleigh numbers considered (up to $10^{10}$ ), the numerical scheme using artificial time-evolution did evolve to a steady state, and that the final steady state was independent of different initial conditions (as in $\S 4.1$ ). In separate calculations with the left-hand side of (2.7) replaced by $\operatorname{Pr}^{-1} \mathrm{D} \boldsymbol{u} / \mathrm{D} t$, it was found that the solutions for a shear-free top also became steady for a sufficiently large but finite Prandtl number $\left(P r=10^{2}\right.$ was sufficient for $\left.R a=10^{9}\right)$. This suggests that these numerical steady states are also likely to be physically stable. Simulations for a rigid top at finite Prandtl number did not evolve to a steady state for $R a \geqslant 3 \times 10^{8}$, even for $\operatorname{Pr}=10^{5}$, which suggests that the infinite-Prandtl-number steady states with a rigid top may not be stable at sufficiently large Rayleigh number. Most of the results presented are for a shear-free top. 
(a)

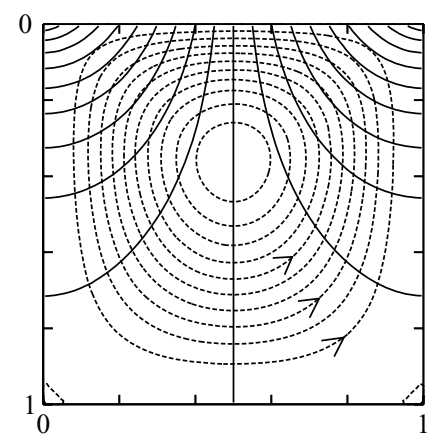

(b)

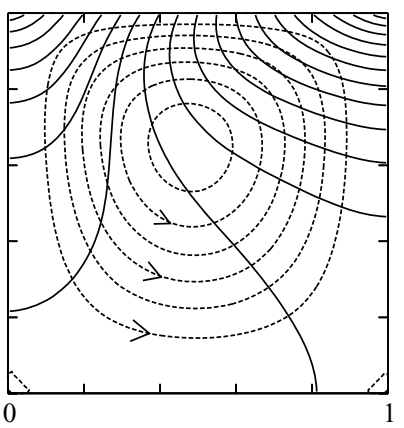

(c)

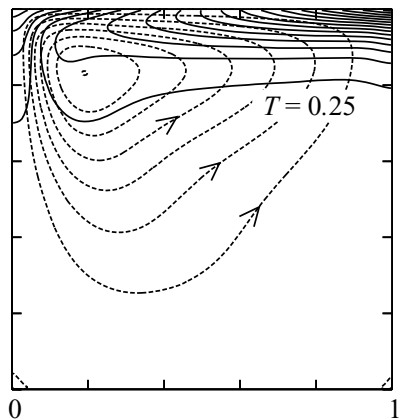

FIGURE 3. Streamlines (dotted) and isotherms (solid) for a shear-free top boundary, with $A=1$ and (a) $R a=1$, (b) $R a=3 \times 10^{3}$, (c) $R a=10^{6}$. The isotherms are plotted at intervals of 0.05 . The intervals between streamlines are $(a) 5 \times 10^{-5},(b) 0.2$, and $(c) 2$. The temperature in the lower half of the box in (c) lies in the range $0.2<T<0.25$, which is much less than the average temperature of 0.5 on the top surface.

\section{Linear and boundary-layer regimes}

It is clear from (2.1) that the strength of the flow and hence the importance of the advection terms in (2.2) increase with $R a$. This gives rise to at least two regimes: at sufficiently small Rayleigh number, advection is negligible, which we term the 'linear' regime; at sufficiently large Rayleigh number, advection is large enough to confine the main thermal variations to a thin boundary layer at the top, which we term the 'boundary-layer' regime. For $A \ll 1$ there is a third regime at intermediate Rayleigh numbers, in which advection is the dominant mechanism of heat transport along the box, but is not strong enough to produce a thermal boundary layer. In $\S 3.1$ we illustrate the two main regimes with numerical results for different aspect ratios. In $\S 3.2$ we derive scalings for the different regimes and the dimensionless parameters that govern the transitions between them. Throughout this section we restrict our attention to a shear-free top boundary. A rigid top gives results that are qualitatively very similar.

\subsection{Qualitative description}

Figure 3 displays examples of the linear and boundary-layer regimes as well as an intermediate stage for a square box $A=1$. For tall boxes $(H \gg L)$ the bottom boundary has only a small effect, and the solutions for $A>1$ are almost the same in the unit square at the top of the box, with a much weaker circulation beneath.

In figure 3(a) the value of $R a$ is sufficiently small that the nonlinear advection terms in (2.2) are unimportant, so the solution is symmetric about the centreline $x=0.5$. The isotherms bend round from the top boundary to satisfy the insulating conditions on the side and bottom walls, and the temperature gradients induce an anticlockwise circulation cell. (A horizontal temperature gradient always produces a circulation, however small the Rayleigh number, in contrast to the existence of a critical Rayleigh number in Bénard convection.)

As $R a$ is increased, the isotherms are deformed by the velocity field, as evident in figure $3(b)$, and the centre of circulation migrates slightly to the left. As $R a$ is increased further, the boundary-layer regime of figure $3(c)$ develops: the main variations in temperature are confined to a near-surface boundary layer, with only 
(a)

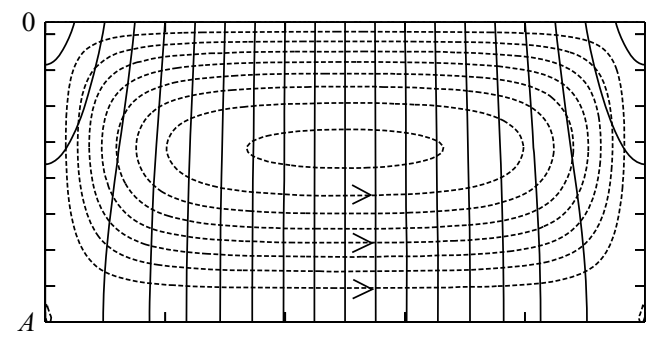

(c)

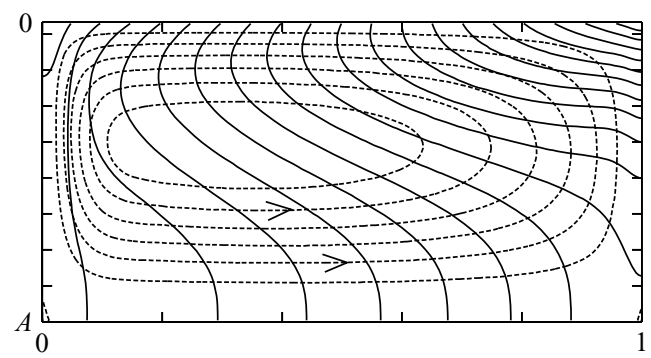

(b)

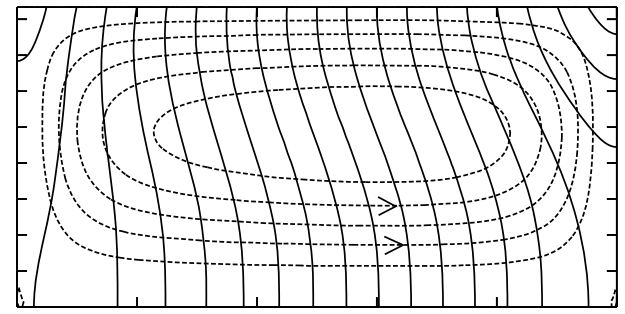

(d)

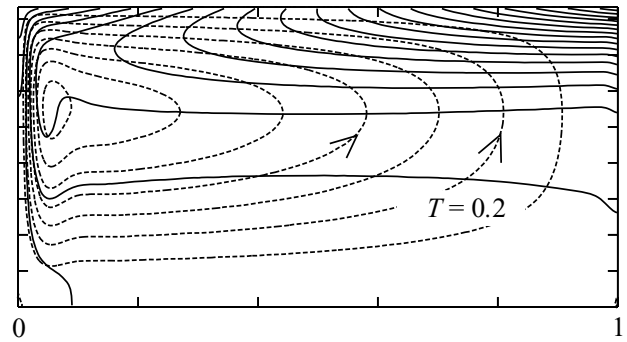

FIGURE 4. Streamlines (dotted) and isotherms (solid) for a shear-free top boundary, with $A=1 / 6$ and (a) $R a=10$, (b) $R a=3 \times 10^{5}$, (c) $R a=3 \times 10^{6}$, and (d) $R a=10^{8}$. The isotherms are plotted at intervals of 0.05 . The intervals between streamlines are $(a) 5 \times 10^{-6},(b) 0.2$, (c) 1 and $(d) 5$.

small variations in the interior. The interior temperature is significantly colder than the average temperature on the top surface.

Figure 4 shows a transition from the linear to boundary-layer regime typical of a shallow box $(H \ll L)$. For small values of $R a$, the isotherms are nearly vertical (figure $4 a$ ), but for larger values of $R a$ the isotherms are deformed by the circulation and the vertical gradient increases (figure $4 b, c$ ). Initially, the isotherms in the lower half of the box are advected further sideways than they are in the upper half, where they are pinned by the thermal boundary condition. The resulting bunching of isotherms in the right half of the box produces a larger vorticity than in the left half, so that, in contrast to the square box, the centre of circulation first moves slightly to the right (figure $4 b$ ). As $R a$ is increased further, a near-surface boundary layer begins to form and the centre of circulation moves back to the left (figure $4 c$ ). In figure $4(d)$ the top boundary layer is well-developed, the centre of circulation is close to the left-hand boundary, and, as in the case $A \geqslant 1$, the temperature variation in the cool interior is much smaller than that in the boundary layer.

\subsection{Scalings in each regime}

\section{Linear regime}

For sufficiently small values of $R a$, leading-order approximations for $T$ and $\psi$ can be found by solving Laplace's equation for $T$ and the inhomogeneous biharmonic equation (2.1) for $\psi$. This leading-order solution can be found, for example, by separation of variables, and clearly $T$ is independent of $R a$ and $\psi$ varyies linearly with $R a$. Hence $N u$ is independent of $R a$ and $P e$ varies linearly with $R a$, in each case with a possible dependence on $A$. 
For $A \geqslant 1$, the bottom boundary has only a small effect on both the near-surface temperatures and the flow, owing to the stronger influence of the sides. Hence

$$
P e \sim R a, \quad N u \sim 1,
$$

independent of $A$.

For $A \ll 1$, Laplace's equation and lubrication theory for a shear-free top boundary yield

$$
T=x, \quad \psi=\frac{A^{4} R a}{48}\left(\frac{z}{A}\right)\left(\frac{2 z}{A}+1\right)\left(\frac{z}{A}-1\right)^{2},
$$

outside an $O(A)$ distance from the ends. To leading order the temperature in this central region is independent of $z$, the vertical heat flux is zero, and the streamfunction is independent of $x$.

The simplest way to evaluate the Nusselt number is to note that the conductive heat flux along the box due to the gradient in (3.2a) is $A$. Owing to the insulating boundary conditions on the sides and bottom, this flux must enter and leave near the ends so that the unsigned Nusselt number (2.6) is given by

$$
N u=2 A \text {. }
$$

In more detail, the asymptotic temperature near the ends for $A \ll 1$ can be found by solving Laplace's equation in a semi-infinite strip with boundary conditions $T_{x}=0$ at $x=0, T \rightarrow x$ as $x \rightarrow \infty, T=x$ at $z=0$ and $T_{z}=0$ at $z=A$. The solution has $\partial T / \partial z \sim \partial T / \partial x \sim 1$ in $x=O(A)$ and the surface heat flux integrates to give (3.3) as expected.

A nonlinear thermal boundary condition $T=T_{0}(x)$ on the top surface produces a temperature field of the form $T=T_{0}(x)+A^{2} T_{1}(x, z)+O\left(A^{4}\right)$ in the central region, where $T_{1}=(z / A)(1-z / 2 A) T_{0 x x}$. The correction $T_{1}$ provides an $O(A)$ vertical heat flux in the central region which must be added to the contribution from the ends to obtain

$$
N u=A\left(\left|T_{0 x}(0)\right|+\int_{0}^{1}\left|T_{0 x x}\right| \mathrm{d} x+\left|T_{0 x}(1)\right|\right) .
$$

Intermediate regime, $A \ll 1$

We turn our attention back to the case of a linear surface condition $T_{0}=x$. The argument leading to (3.3) assumed that conduction is the dominant mode of heat transfer. For $A \ll 1$ it is possible for vertical conduction to maintain (3.2a) as the leading-order temperature distribution, but for advection by the induced flow (3.2b) to give the leading-order heat flux from one end to the other rather than horizontal conduction.

Since there is no net horizontal mass flux, advection of the leading-order temperature field $T=x$ produces no net transport of heat. It does, however, advect the isotherms at the bottom to the right, producing a perturbation

$$
\delta T=-\frac{A^{5} R a}{48 \times 20} \frac{z^{2}}{A^{2}}\left(10-15 \frac{z^{2}}{A^{2}}+8 \frac{z^{3}}{A^{3}}\right) .
$$

(The isotherms at the top are pinned to the surface condition.) Because $\delta T$ is more negative at the bottom than the top, advection of $\delta T$ does give a net heat flux along the box, and leads to the result

$$
N u \sim 2 \int_{0}^{A} \psi_{z} \delta T \mathrm{~d} z=\frac{19 A^{9} R a^{2}}{315 \times 48^{2}} .
$$


The advected flux enters and leaves the box near the ends. In this regime the velocities are sufficiently large that the adjustment to the end boundary conditions occurs not over the $O(A)$ length scale of the linear conductive regime but over the $O\left(A^{5} R a\right)$ length scale of the thermal entry length of the $O\left(A^{3} R a\right)$ velocity in a channel of height $A$. This thermal entry length lies between the height and length of the box if $A \ll A^{5} R a \ll 1$. The advected flux (3.6) dominates the linear conductive flux (3.3) if $A \ll A^{9} R a^{2}$. The perturbation (3.5) is smaller than the background linear gradient $T=x$ if $A^{5} R a \ll 1$. Under the same condition, the end adjustment regions are short enough for (3.5) to hold over most of the box. These considerations all suggest that this intermediate regime is valid for $A \ll A^{5} R a \ll 1$.

\section{Boundary-layer regime}

For sufficiently large values of $R a$, temperature variations will be confined by a strong flow to a top thermal boundary layer of some thickness $\delta \ll 1$. For a rigid boundary it is natural to assume that the characteristic vertical scale of the velocity variation below the boundary is also $\delta$. For a shear-free boundary this assumption of equal vertical scales is less obvious, but nevertheless still valid. Using this assumption, and noting that $T \sim x \sim 1,(2.1)$ and (2.2) yield $\psi / \delta^{4} \sim R a$ and $\psi \sim 1 / \delta$ respectively. Solving these two balances gives

$$
\psi \sim R a^{1 / 5}, \quad \delta \sim R a^{-1 / 5},
$$

as suggested by Rossby (1965). From (3.7b) and (2.6), we also obtain

$$
N u \sim R a^{1 / 5} .
$$

For $A \ll 1$ the requirement that $\delta \ll A$ implies that $A^{5} R a \ll 1$.

We shall return to the assumption of equal vertical scales in $\S 4.2$. For now, we note that it is consistent with the numerical results of figures $3(c)$ and $4(d)$, which show the centre of circulation lying within the thermal boundary layer.

\subsection{Numerical transitions between regimes}

Case $A \geqslant 1$

For $A \geqslant 1$, the bottom boundary has only a small effect on both the region of strongest flow and the near-surface temperatures, and hence it is simply $R a$ that governs the transition between the linear and boundary-layer regimes. Hence, a universal dependence of $P e$ and $N u$ on $R a$ is expected for different values $A \geqslant 1$. This universal dependence is shown by the collapse of the curves for $A=1$ and $A=2$ in figures 5 and 6 ; the 'large- $A$ ' behaviour extends remarkably well down to $A=1$. The curves confirm the scalings (3.1) in the linear regime, and move towards the 1/5-power laws of (3.7a) and (3.8) in the boundary-layer regime. In $\S 4.2$ we consider larger Rayleigh numbers for better evidence of the $1 / 5$-power law for $A \geqslant 1$. The transition between linear and boundary-layer regimes occurs over roughly a decade on either side of $R a=10^{3}$.

\section{Case $A \ll 1$}

In $\S 3.2$ we saw that for $A \ll 1, A^{5} R a \ll 1$ is required for the leading-order flow and temperature to be given by (3.2), and $A^{5} R a \gg 1$ for the boundary-layer thickness $\delta$ to be much less than $A$. Hence $A^{5} R a$ governs the transition between the intermediate and boundary-layer regimes. The strength $P e=\psi_{\max }$ of the flow has the same scalings in the linear and intermediate regimes. Hence, from (3.2b) and (3.7a), we expect a universal curve of $A P e$ against $A^{5} R a$ for $A \ll 1$ that moves from a linear to a 


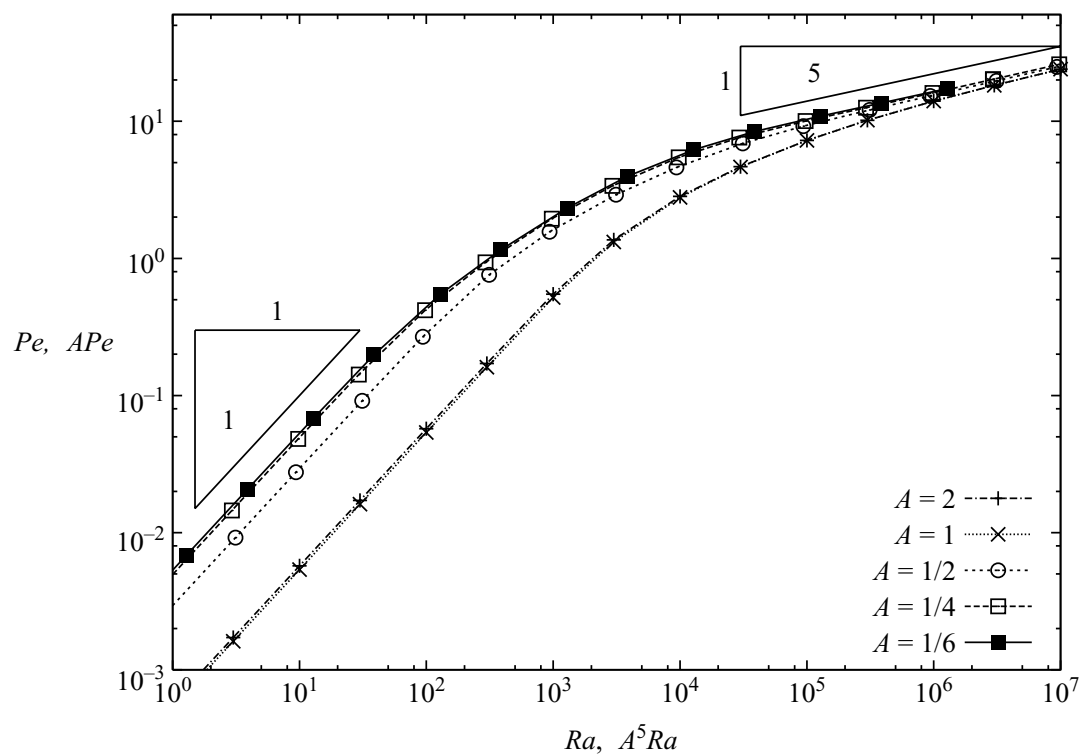

Figure 5. Strength of circulation $P e$ against $R a$ for $A \geqslant 1$ and $A P e$ against $A^{5} R a$ for $A<1$, for a shear-free top boundary. The linear and intermediate regimes both give slope 1 , and the boundary-layer regime gives slope $1 / 5$.

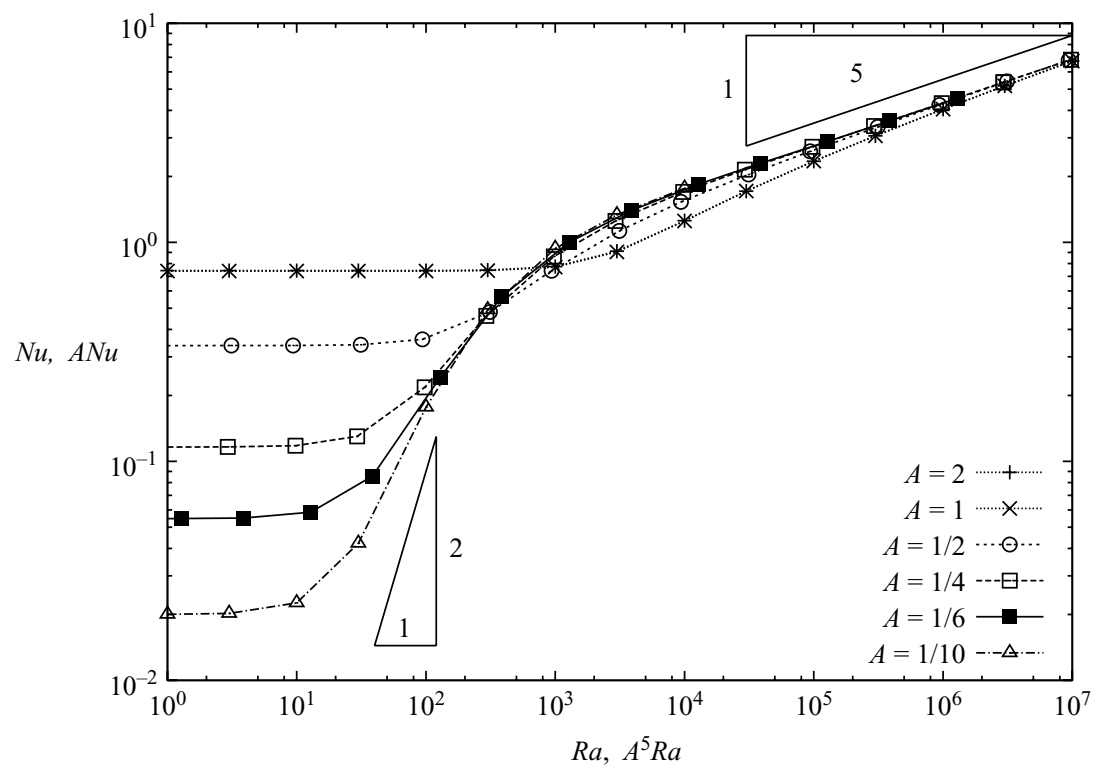

FiguRE 6. Heat transfer $N u$ against $R a$ for $A \geqslant 1$ and $A N u$ against $A^{5} R a$ for $A<1$, for a shear-free top boundary. The boundary-layer regime gives slope $1 / 5$, and the linear and intermediate regimes are distinct for $A \ll 1$.

1/5-power law as $A^{5} R a$ increases. This is confirmed in figure 5, with the results for $A=1 / 2$ not quite collapsing onto those for the smaller values of $A$.

In figure 6 we plot $A N u$ against $A^{5} R a$ to illustrate the transition to the boundarylayer regime. The 1/5-power-law scaling of (3.8) is confirmed for large values of

\section{CAmbridge JDUNALS}




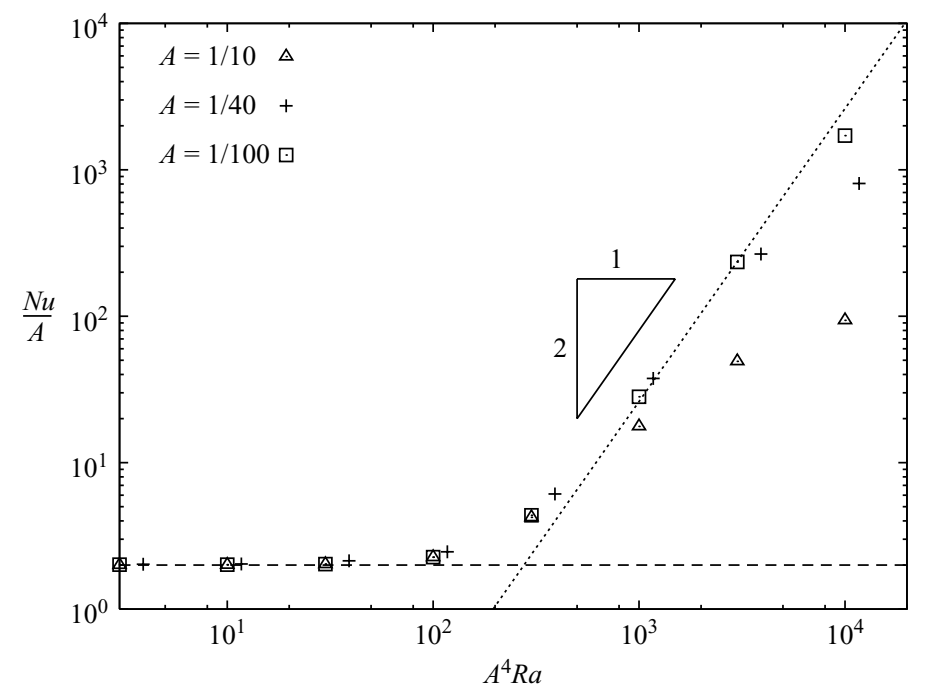

FIGURE 7. Heat transfer $N u / A$ against $A^{4} R a$ for $A \ll 1$, for a shear-free top boundary. In the linear regime the heat flux asymptotes to $N u / A=2$ (dashed line), and in the intermediate regime it asymptotes to $N u / A=19\left(A^{4} R a\right)^{2} /\left(315 \times 48^{2}\right)$ (dotted). See text for details.
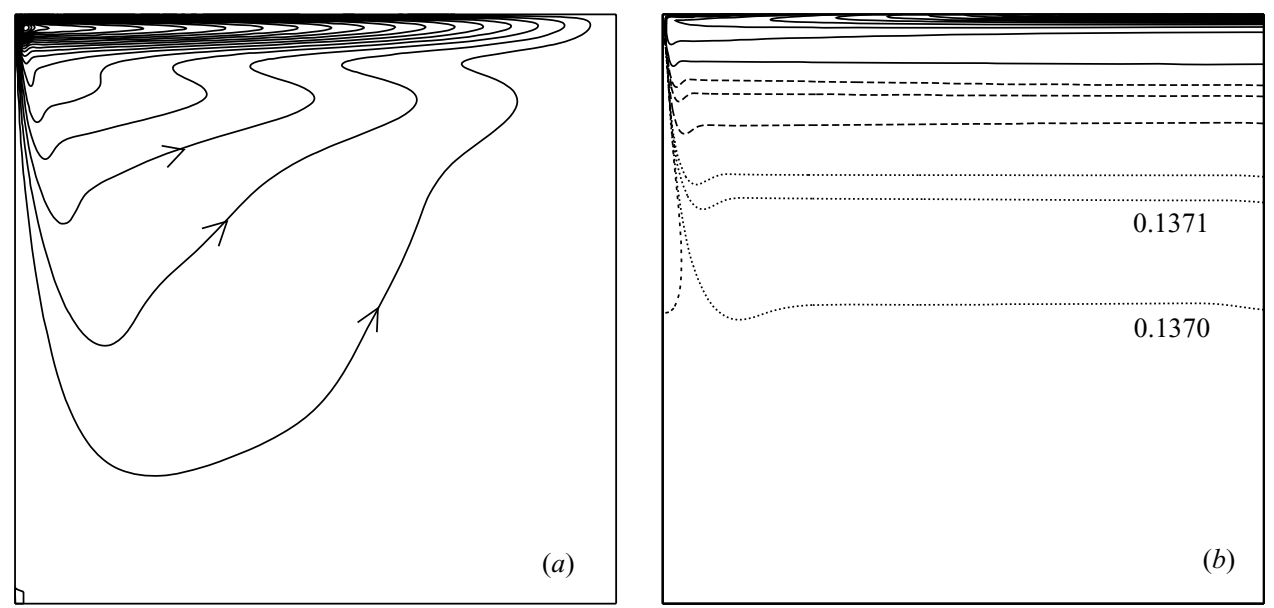

FIGURE 8. (a) Streamlines for $R a=10^{10}, A=1$ and a shear-free top boundary. The interval between contours is 5. (b) Corresponding isotherms with maximum level, minimum level, increment as follows: 0.95, 0.15, 0.05 (solid); 0.142, 0.138, 0.002 (long dashed); 0.1372, 0.1370, $10^{-4}$ (dotted); 0.13698 (dashed). The temperature in the bottom right corner is 0.136989 .

$A^{5} R a$. In figure 7 we plot $N u / A$ against $A^{4} R a$ for very small values of $A$ to illustrate the transition between the linear and intermediate regimes. This plot also confirms the numerical coefficients in (3.3) and (3.6).

\section{Asymptotic structure in the large- $R a$ regime}

As an introduction to the asymptotic structure, the steady solution for $R a=10^{10}$, $A=1$ and a shear-free top, is shown in figure 8. At such a large value of $R a$, the top boundary layer is extremely thin, and a stretched grid is employed in the numerical

\section{CAMBridge JDURNALS}



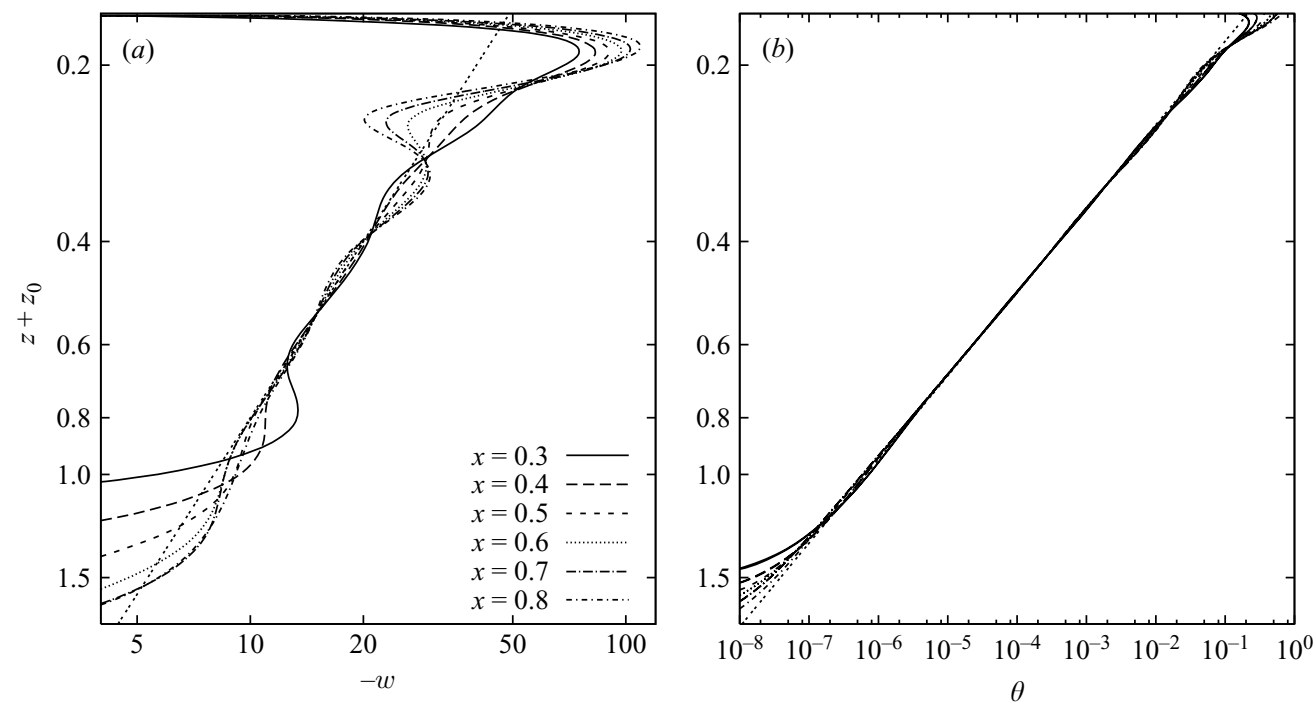

FiguRE 9. Vertical profiles at different horizontal positions $x$ of $(a)$ the upwards velocity $-w$ and $(b)$ the temperature difference from the bottom temperature $\theta=T-T_{b}$, with $R a=10^{10}$ and $A=2$. The straight lines are respectively $-w=8 /\left(z+z_{0}\right)$ and $\theta=c / \operatorname{Ra}\left(z+z_{0}\right)^{7}$, with $z_{0}=4.38 / R a^{1 / 7}$ and $c=6610($ see $\S 4.3)$.

calculations to give full resolution of the boundary layer and the flow in the top left corner. The streamlines (figure $8 a$ ) show that most of the return flow comes back near the top surface, and only a small part descends the left-hand wall to feed a much weaker flow through the interior. It is also noticeable that this interior flow oscillates from side to side as it rejoins the top boundary layer.

The isotherms (figure $8 b$ ) are plotted using a variable spacing to show some of those running through the interior. The main temperature variations are strongly concentrated in the top boundary layer, with only small temperature variations in the interior. One can see from the near-horizontal isotherms in the interior that stratification dominates the flow there, i.e. the interior is strongly stratified in the sense of having a strong dynamical effect. However, as can be seen by the isotherm values, the magnitude of the stratification decreases very rapidly with depth, with the variations in the bottom half of the box less than about $10^{-6}$. The almost uniform temperature $T_{b}$ at depth, say at the bottom right corner, is about 0.137 , which is much less than the mean temperature 0.5 of the top boundary.

The structure of the interior is revealed more clearly by logarithmic profiles of the vertical velocity $w$ and temperature difference, $\theta=T-T_{b}$, where $T_{b}$ is the bottom temperature. Figure 9 shows vertical profiles at different horizontal positions $x$ in a tall box with $A=2$ and $R a=10^{10}$. The origin of the vertical axis is offset by $z_{0}=4.38 / R a^{1 / 7}$ for reasons explained in $\S 4.3$. Figure $9(a)$ shows that the upwelling $-w$ is not quite uniform, but is nevertheless approximately given by $8 /\left(z+z_{0}\right)$. The deviations of $-w$ from $8 /\left(z+z_{0}\right)$ are of three types. Deviations in $z+z_{0}<0.25$ are associated with the structure of entrainment into the top boundary layer; those in $z+z_{0}>0.8$ are mainly associated with the descending plume on the left-hand wall, which occupies a noticeable width of the box at this depth; in $0.25<z+z_{0}<0.8$ there is a stack of weak counter-rotating cells superposed on the uniform upwelling, with the profiles for different $x$ crossing each other at the boundaries between cells. 


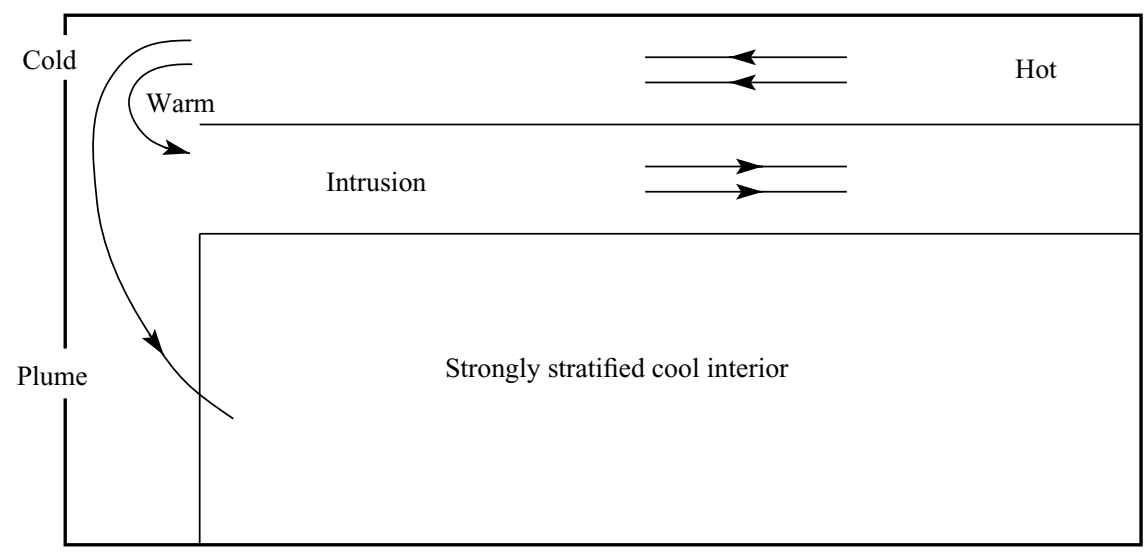

FIGURE 10. Separation of the return flow into a warm shallow intrusion and a much weaker cold wall plume. The plume detrains into the cool interior and maintains its stratification. Although the temperature variations in the cool interior are small in magnitude, the stratification is strong in its dynamical effect and confines most of the return flow to the shallow intrusion.

Figure $9(b)$ shows that the temperature is indeed strongly stratified in the interior, with very small horizontal variations in temperature and a vertical decay of $\theta$ through the interior proportional to $\left(z+z_{0}\right)^{-7}$. In $z+z_{0}>1.2$, the temperature difference $\theta$ decays a little more rapidly owing to the choice of reference temperature $T_{b}$.

In the rest of this section we explain the structure of the flow, and the scalings and interaction of the different regions. In $\S 4.1$ we explain how the return flow exiting the top left corner splits into a warm horizontal intrusion and a cold downwelling plume, and consider transients of numerical simulations to support these ideas. In $\S 4.2$ we consider the top boundary layer, and explain that the $R a^{1 / 5}$ scalings of (3.7) are independent of boundary condition because of the strong shear in the boundary layer from the shallow intrusion. In $\S 4.3$ we find a similarity solution for the downwelling plume, and derive the solutions for the interior flow and temperature that were plotted above. We also examine the cellular structure and corner region, and discuss the matching between these regions and the plume and interior. In $\S 4.4$ we examine the dependence of the interior temperature on the imposed boundary conditions, and consider the effect of aspect ratio.

\subsection{Evolution to stratification and a shallow intrusion}

Figure 8 suggests that the stratified cool interior forces most of the return flow back in a shallow intrusion, in which the fluid is warmer than the interior underneath, but cooler than the top boundary layer. A small portion of the return flow, which has been strongly cooled by the left-most part of the top boundary, descends into the box as a cold downwelling plume on the left-hand wall, and then detrains into the cool interior before returning slowly up into the top boundary layer. The side plume and interior therefore constitute a viscous 'filling box', in which the plume maintains the interior stratification. This structure of the return flow is shown schematically in figure 10 .

These dynamical ideas were explored further by studying the transients in the numerical evolution to a steady state. For this purpose, rather than use the artificial time-stepping of (2.7), we included inertial terms so that the left-hand side of (2.7) 
(a)

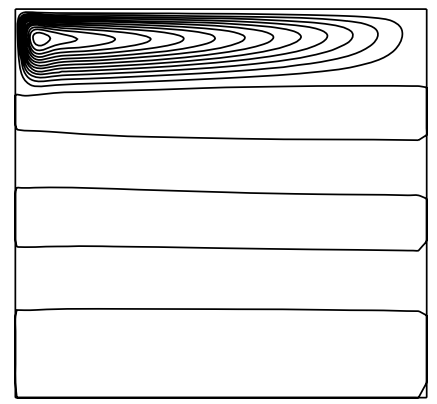

(b)

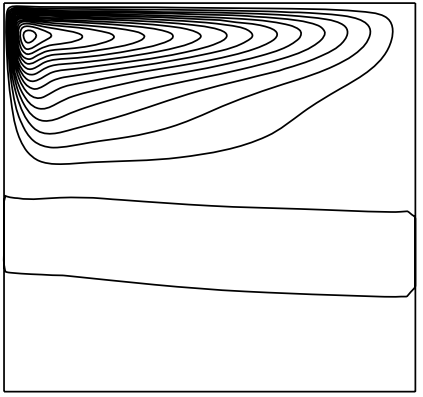

(c)

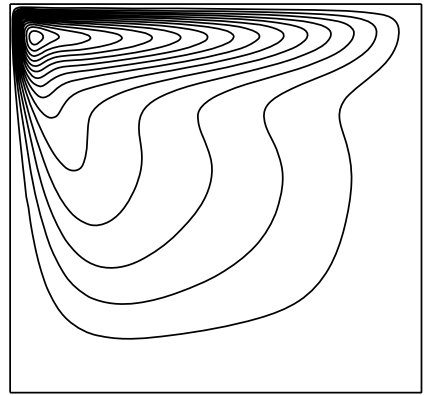

FiguRE 11. Streamlines at stages in the evolution to steady state for a rigid top boundary with $R a=10^{8}$ and $A=1$, and $\operatorname{Pr}=10^{3}$, and an initial temperature of 0 . The time elapsed (see figure $13 a$ ) is: $(a) 0.1,(b) 1,(c) 2.5$. The interval between contours is 2 .

(a)

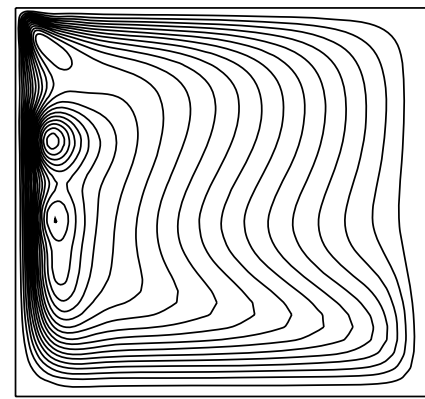

(b)

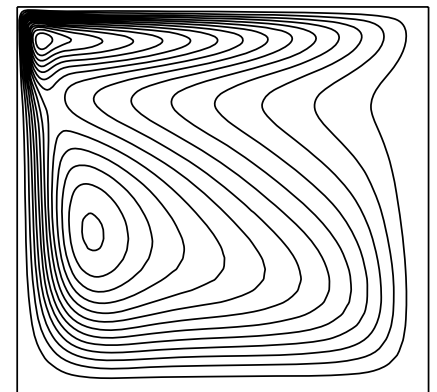

(c)

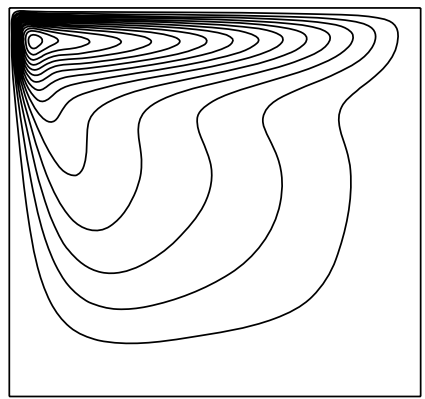

FIGURE 12. Parameters as for figure 11, but with an initial temperature of 0.5 . The time elapsed (see figure 13b) is: $(a) 0.03,(b) 0.3,(c)$ 1.2. The interval between contours is 5 for $(a)$, and 2 for $(b)$ and $(c)$.

is replaced by $\operatorname{Pr}^{-1} \mathrm{D} \boldsymbol{u} / \mathrm{D} t$ (where the time scale is non-dimensionalized with $L^{2} / \kappa$ ). For illustration we used $\operatorname{Ra}=10^{8}, A=1$ and $\operatorname{Pr}=10^{3}$.

In figure 11 we show transients from a calculation with a rigid top boundary in which the initial temperature of the fluid was set to 0 , the minimum temperature on the top boundary. Initially the flow is characterized solely by a top boundary layer and shallow intrusion (figure 11a), as the return flow is all warmer than the cold interior and cannot sink. Diffusion of heat through the depth of the fluid slowly warms the interior and a plume begins to develop on the left-hand wall (figure $11 b$ ). The steady state (figure $11 c$ ) is reached when the interior has warmed sufficiently and the plume developed sufficiently that the downwards diffusion of heat from the top boundary layer is balanced by the supply of cold fluid in the plume.

In figure 12 we show transients from a calculation in which the initial temperature of the fluid was set to 0.5 , the average temperature imposed on the top boundary. Here the evolution to steady state is significantly more complex, but as in the previous calculation we also observe a competition between the plume and intrusion for the return flow. In figure 12(a) the flow is characterized by a strong downwelling plume on the left-hand wall, due to the colder part of the top boundary. The plume carries cold fluid into the interior, thus cooling it. Consequently the temperature difference between the plume and interior decreases, which weakens the plume. Some of the 
(a)

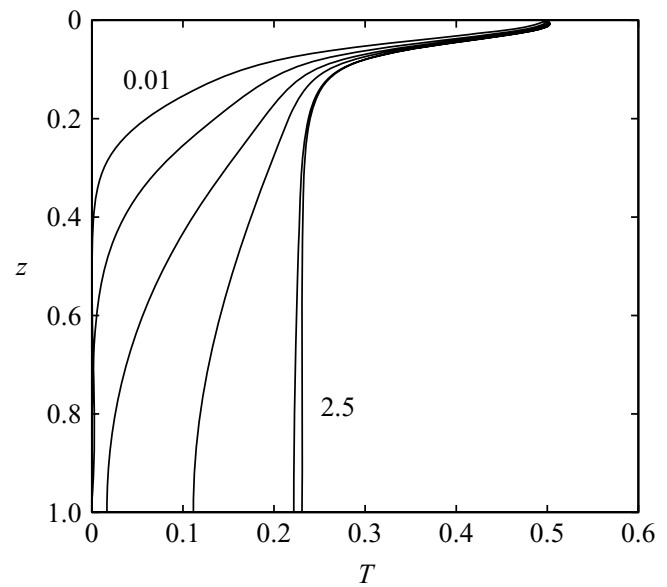

(b)

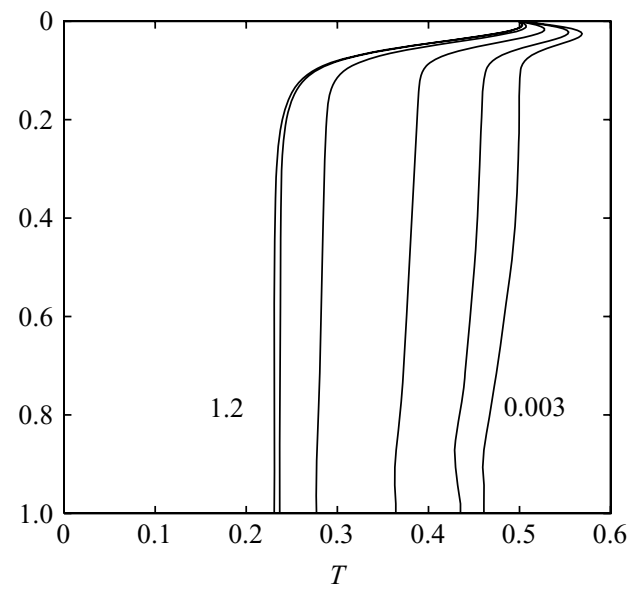

Figure 13. Temperature profile at $x=0.5$ in the evolution to steady state for $(a)$ an initial temperature of 0 , and (from left to right) $t=0.01,0.03,0.1,0.3,1$ and $2.5 ;(b)$ an initial temperature of 0.5 , and (from right to left) $t=0.003,0.01,0.03,0.1,0.3$ and 1.2. In both cases, $R a=10^{8}, A=1$ and $\operatorname{Pr}=10^{3}$.

warmer fluid starts to return in a shallow intrusion and the top boundary layer develops (figure 12b). The steady state (figure 12c) is the same as before, and is reached when the interior has cooled sufficiently and the plume weakened sufficiently that downwards diffusion of heat from the top boundary is able to balance the further supply of cold fluid in the plume. Most of the return flow then occurs in the intrusion.

Figure 13 shows the evolution of the vertical temperature profile at the mid-section $x=1 / 2$ for each of the two calculations whose streamlines are shown in figures 11 and 12. For the initial temperature of 0 , the profiles in figure 13(a) illustrate the slow diffusion from the top boundary layer, which leads to an $O(1)$ time scale for the evolution to steady state in these calculations with $A=1$. (Presumably, it would be $O\left(A^{2}\right)$ if $A \ll 1$.) For the initial temperature of 0.5 , figure $13(b)$ shows that the time taken to reach steady state is significantly less than the $O(1)$ diffusive time scale. It is also much greater than the very short overturning time scale for the strong flow in figure 12(a). The temperature below the top boundary layer is nearly uniform with depth, consistent with more rapid overturning than evolution to steady state, and is also (though not visible in the figure) nearly uniform across the box away from the plume.

The evolution described above for a cold initial temperature (figure 11) was observed experimentally by Mullarney et al. (2004), who argued for a similar mechanism in the approach to the final state. Evolution from both cold and hot initial temperatures was investigated experimentally by Wang \& Huang (2005).

\subsection{Horizontal boundary layer and return flow of the shallow intrusion}

For a rigid boundary, the boundary-layer flow is driven along the top boundary like a viscous gravity current (Huppert 1982) by the horizontal gradient of the hydrostatic pressure. Owing to the no-slip boundary condition, the dominant shear occurs within the boundary layer and hence the characteristic scales for the velocity and temperature gradients are the same. This was the assumption used to derive the $R a^{1 / 5}$ scalings of (3.7). 

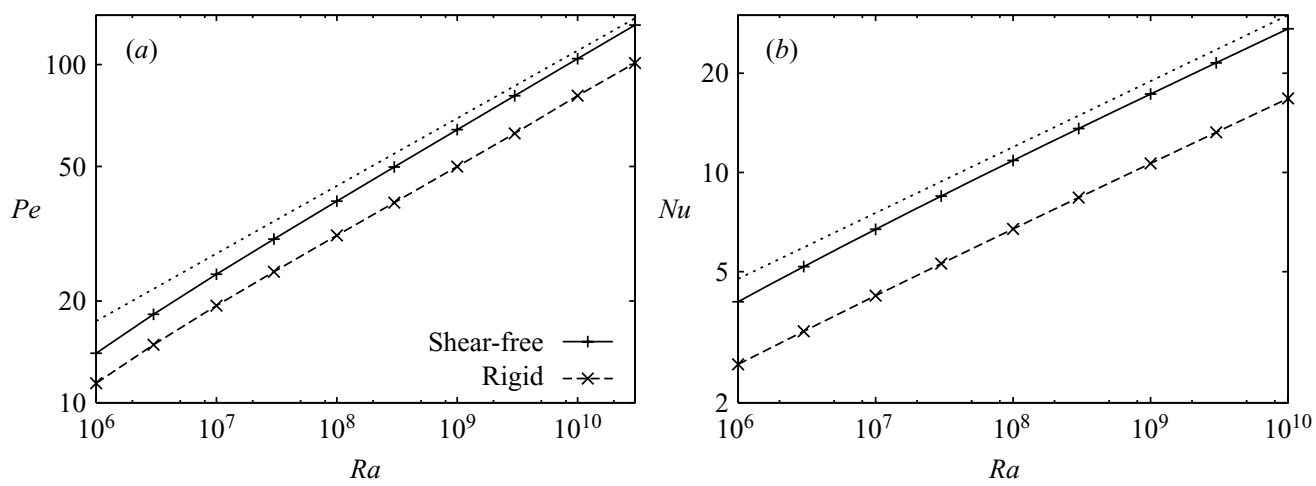

Figure 14. (a) $P e$ and (b) $N u$ against $R a$ for $A=1$ in the boundary-layer regime. The asymptotes (dotted) in both plots have slope $1 / 5$.

For a shear-free boundary, it is less obvious that the scalings (3.7) still hold, and indeed these scalings have been called into question (e.g. Mullarney et al. 2004; Siggers et al. 2004; Gramberg et al. 2007).

A standard assumption with free-surface boundary layers is that the velocity in the boundary layer is to leading-order uniform, and given by matching to a larger-scale flow further from the boundary. In the absence of a no-slip condition, any horizontal pressure gradient in the boundary layer must be supported by the shear stress in the interior, rather than the shear stress against the boundary (Roberts cf. 1977; Lister \& Kerr 1989; Davis, Schonberg \& Rallison 1989). For a boundary-layer thickness of $\delta$, this shear stress $\psi_{z z} \sim R a \delta^{2}$ from (2.1), since $T_{x} \sim 1$. If we were to assume that the return flow is spread over the depth of the box (with $A=1$ for simplicity), then $z \sim 1$ in $\psi_{z z}$ and hence the interior streamfunction $\psi_{i} \sim R a \delta^{2}$. Then in the boundary layer $\psi \sim R a \delta^{3}$ and from (2.2) $\psi \sim 1 / \delta$. Assuming a boundary layer with a return flow over the depth of the box would therefore lead to the scalings $\psi \sim R a^{1 / 4}, \delta \sim R a^{-1 / 4}$, and $P e \sim N u \sim R a^{1 / 4}$ rather than $R a^{1 / 5}$. Gramberg et al. (2007) developed an asymptotic theory based on these scalings with $A \ll 1$, to obtain an ordinary differential equation for the boundary-layer velocity.

The problem with this scaling argument is the assumption that the return flow occurs over the depth of the box, which leads to an incorrect scaling for the velocity in the boundary layer. As discussed in $\S 4.1$, the interior stratification confines most of the return flow to a shallow intrusion of comparable thickness to the top boundary layer, and hence $\psi_{i} \sim R a \delta^{4}$ not $R a \delta^{2}$. The intrusion means that there is strong shear in the boundary layer even for a shear-free boundary condition, invalidating the assumption of a uniform-velocity boundary layer. As in the case of a rigid boundary condition, the characteristic scales for velocity and temperature gradients are the same for shear-free conditions, and hence we again obtain (3.7).

The numerical results confirm that the Péclet and Nusselt numbers indeed scale as $R a^{1 / 5}$ for both rigid and shear-free conditions (figure 14), though in the case of Péclet number and the shear-free condition, very large Rayleigh numbers $\left(>10^{8}\right)$ are required to reach the asymptotic slope. Even more clearly, the numerics refute any suggestion of $R a^{1 / 4}$ scalings from the usual free-surface boundary-layer assumptions, and show that the $R a^{1 / 3}$ estimate of Siggers et al. (2004) is only a weak bound. (Also, the $R a^{1 / 4}$ scalings will not apply in an intermediate regime, as hypothesized by Gramberg et al.; the discussion of the transition between the linear and 


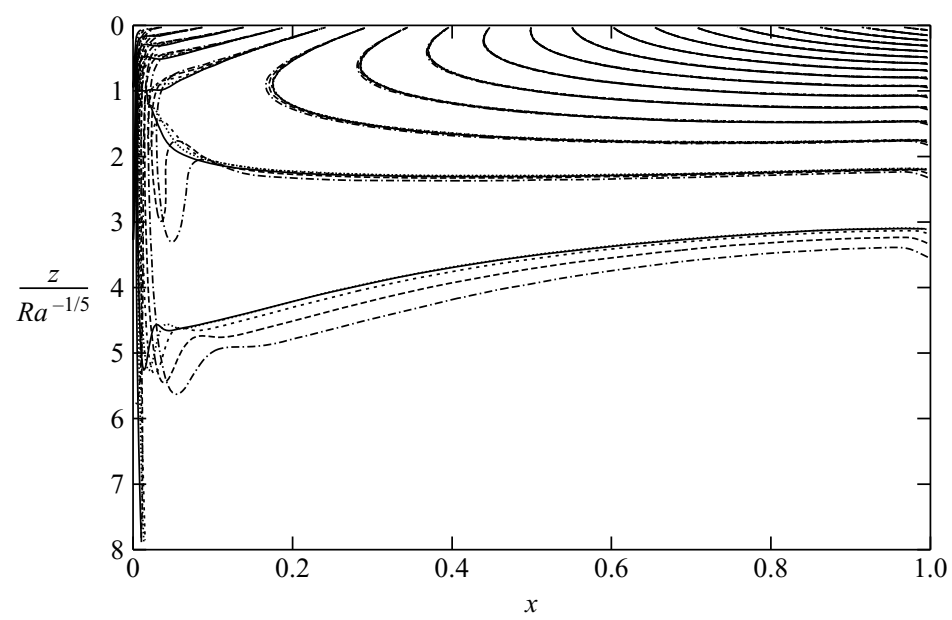

FIGURE 15. Collapse of isotherms in the boundary layer for a shear-free top with $A=2$, and $R a=10^{8}$ (dot-dash), $3 \times 10^{8}$ (long dashed), $10^{9}$ (dashed), $3 \times 10^{9}$ (dotted) and $10^{10}$ (solid). The isotherms are plotted in 0.05 increments, and the lowest horizontal isotherm has value 0.2 .

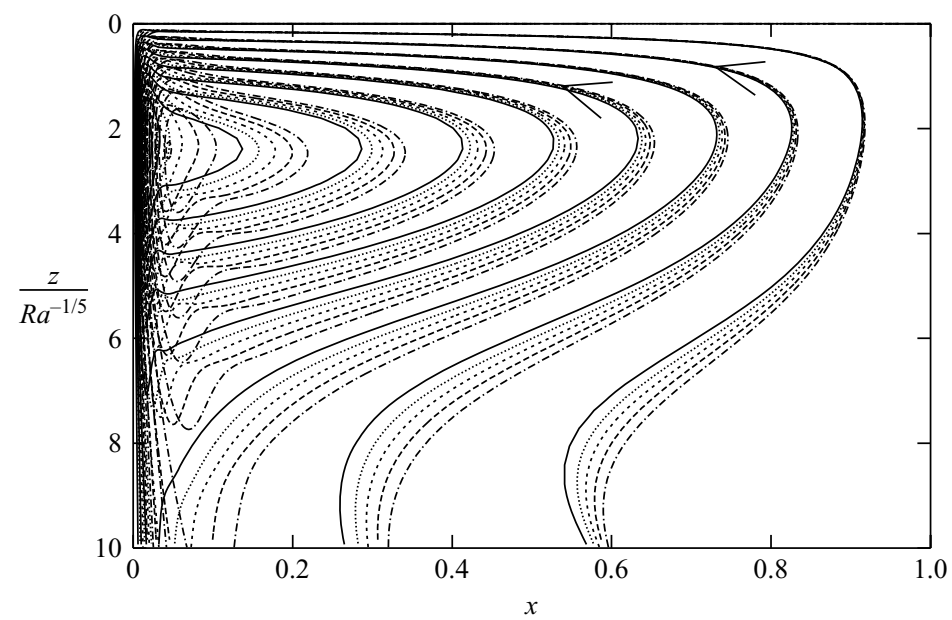

FIGURE 16. Collapse of scaled streamlines $\psi / R a^{1 / 5}$ in the boundary-layer for a shear-free top at $A=2$, and $R a=10^{8}$ (dot-dash), $3 \times 10^{8}$ (long dashed), $10^{9}$ (dashed), $3 \times 10^{9}$ (dotted) and $10^{10}$ (solid). The streamlines are plotted in 0.1 increments.

boundary-layer regimes in $\S 3.2$ gives a complete characterization of the scalings in the parameter space $(A, R a)$.)

We also compare the results for rigid and shear-free boundary conditions in figure 14. The shear-free case leads as might be expected to a larger volume flux than the rigid case, by approximately $25 \%$ (figure $14 a$ ). The Nusselt number is approximately $60 \%$ greater for the shear-free case (figure $14 b$ ), presumably because the flow for the shear-free condition leads to a larger deformation of the isotherms, and hence to larger vertical temperature gradients.

We can use the boundary-layer scalings to collapse the isotherms and streamlines for different Rayleigh numbers. In figure 15, the isotherms are collapsed over two decades of Rayleigh number with the vertical scale $z / R a^{-1 / 5}$. The corresponding scaled streamlines $\psi / R a^{1 / 5}$ are shown in figure 16 . The collapse of both the 
left-moving boundary layer and the right-moving intrusion is excellent (figure 16). Most of the return flow, approximately $70 \%$ over the two decades of Rayleigh numbers shown, occurs in the intrusion within a depth $z \approx 8 R a^{-1 / 5}$ and does not pass into the interior.

\subsection{Plume and strongly stratified interior}

\section{Interior and plume equations}

We now turn our attention to an analysis of the plume and interior flow. As shown schematically in figure 10, some of the fluid falls down the left-hand side in a cold downwelling plume, which then detrains over a range of heights into a stratified interior. The following analysis applies to intermediate heights, $R a^{-\alpha} \ll z \ll 1$, where we argue later that $\alpha=1 / 7$. This region lies beneath the near-surface structure of the boundary layer and intrusion, and is sufficiently far from the bottom that the side plume is much narrower than the width of the interior. From figures 8 and 9, we note that $T_{x} \ll T_{z}$ in the interior, so that we can write $T=T_{0}(z)+T_{1}(x, z)$, with $T_{1} \ll T_{0}$. We also note that $\psi_{x}$ is not much larger than $\psi_{z}$ in the interior. Hence, as verified below, we assume that $\psi_{z} T_{1 x} \ll \psi_{x} T_{0 z}$ and deduce that the leading-order balance in (2.2) is

$$
w T_{0 z}=T_{0 z z}
$$

i.e. upwards advection of cold fluid balances downwards diffusion of heat, as discussed regarding the transient numerical simulations in $\S 4.1$. We deduce from $(4.1 a)$ that the upwelling velocity $-w$ in the interior is independent of $x$ at leading order, in agreement with the main trend at intermediate heights in figure 9. From the no-flux condition at $x=1$ it follows that at leading order in the interior

$$
\psi=-w(z)(1-x) .
$$

(The no-slip boundary condition at $x=1$ is satisfied by a weak, dynamically unimportant boundary layer, driven by a small perturbation to the horizontal stratification.)

Since $\partial / \partial x \gg \partial / \partial z$ in the plume, we apply the lubrication approximation to $(2.1)$ and (2.2), giving

$$
\psi_{x x x x}=R a T_{x}, \quad-\psi_{z} T_{x}+\psi_{x} T_{z}=T_{x x} .
$$

Detrainment of the plume into the interior is described by matching $\psi$ and $T$ from the plume approximation (4.2) to the interior approximation (4.1).

Solution for the interior and similarity solution for the plume

In the plume it is natural to seek a similarity solution in which $x \sim\left(z+z_{0}\right)^{p}$ and $\theta=T-T_{b} \sim\left(z+z_{0}\right)^{-q}$ for some exponents $p$ and $q$ (see Worster \& Leitch 1985), where we have included a vertical offset $z_{0}$ allowed by the equations and assumed that $T \rightarrow T_{b}$ at depth. From $(4.1 a),-w=(q+1)\left(z+z_{0}\right)^{-1}$, and then from scaling (4.2), $p=2$ and $q=7$. In the interior we therefore have

$$
\psi=\frac{8(1-x)}{z+z_{0}}, \quad \theta=\frac{c}{R a\left(z+z_{0}\right)^{7}},
$$

for some constant $c$, to be determined by matching onto the solution for the plume. We note that this solution must break down for $z+z_{0} \ll R a^{-1 / 7}$ since $\theta$ can be at most $O(1)$. For $R a^{-1 / 7} \ll z+z_{0} \ll 1$ the leading-order interior flow,

$$
\boldsymbol{u}=\left(\frac{8(1-x)}{\left(z+z_{0}\right)^{2}}, \frac{-8}{z+z_{0}}\right),
$$


is driven across the box by a small tilt of the isotherms,

$$
R a T_{1 x}=\nabla^{4} \psi=\frac{192(1-x)}{\left(z+z_{0}\right)^{5}} \ll R a T_{0 z}=\frac{-7 c}{\left(z+z_{0}\right)^{8}},
$$

away from perfect horizontal stratification. As anticipated, $\psi_{z} T_{1 x} \ll \psi_{x} T_{0 z}$.

In the plume we now seek a similarity solution

$$
\psi=\frac{F(\eta)}{z+z_{0}}, \quad \theta=\frac{G(\eta)}{R a\left(z+z_{0}\right)^{7}}, \quad \text { where } \quad \eta=\frac{x}{\left(z+z_{0}\right)^{2}},
$$

with $F \rightarrow 8$ and $G \rightarrow c$ as $\eta \rightarrow \infty$ from (4.3) Equation (4.2) becomes

$$
F^{\prime \prime \prime}=G-c, \quad F G^{\prime}-7 F^{\prime} G=G^{\prime \prime},
$$

where a prime denotes differentiation with respect to $\eta$, which can be rewritten as

$$
F^{(\mathrm{v})}-F F^{(\mathrm{iv})}+7 F^{\prime}\left(F^{\prime \prime \prime}+c\right)=0 .
$$

We can scale out $c$ with

$$
F=c^{1 / 4} f, \quad \eta=c^{-1 / 4} \xi
$$

to obtain

$$
f^{(\mathrm{v})}-f f^{(\mathrm{iv})}+7 f^{\prime}\left(f^{\prime \prime \prime}+1\right)=0,
$$

where the prime now denotes differentiation with respect to $\xi$.

The boundary conditions for a rigid insulating wall are

$$
f=f^{\prime}=f^{(\mathrm{iv})}=0 \text { at } \eta=0 .
$$

(For a shear-free side $f^{\prime}=0$ is replaced by $f^{\prime \prime}=0$.) To ensure that $F \rightarrow 8$ as $\eta \rightarrow \infty$, we must have $f \rightarrow f_{\infty}$ for some constant $f_{\infty}$, and the constant $c$ is then given by

$$
c=\left(8 / f_{\infty}\right)^{4} \text {. }
$$

Linearizing (4.10) about $f=f_{\infty}$ gives solutions $\exp k \xi$ such that

$$
\left(k^{4}-f_{\infty} k^{3}+7\right) k=0 .
$$

The root $k=0$ corresponds to a small change in $f_{\infty}$. Two of the non-zero roots are in the right-half complex plane $(\operatorname{Re}(k)>0)$ and give growing modes that must be suppessed if $f \rightarrow f_{\infty}$. The other two non-zero roots are in the left-half-plane $(\operatorname{Re}(k)<0)$; if

$$
0<f_{\infty}^{4}<1792 / 27
$$

they are complex and decaying oscillatory behaviour is expected in the far field. The need to suppress two growing modes gives two boundary conditions at infinity, which, together with the three conditions of (4.11), imply a unique solution for $f$ and thence for $c, F$ and $G$. We integrated (4.10) with AUTO97 (Doedel et al. 1997), and obtained $f_{\infty}=0.887, G(0)=-4980$ and $c=6610$ from (4.13).

The only free parameters of the solution for the plume and interior are the offset $z_{0}$ and the temperature $T_{b}$. In the limit $A \rightarrow \infty$ (so that the bottom boundary does not affect the interior), both are determined in principle by matching the plume and interior onto the corner region and top boundary layer. In practice, we determine them from the numerical solutions (see below).

Comparison with numerical solutions

We compare the similarity solution with numerical results, obtained for a tall box $(A=2)$ to limit any effect of the bottom boundary. We can then accurately 


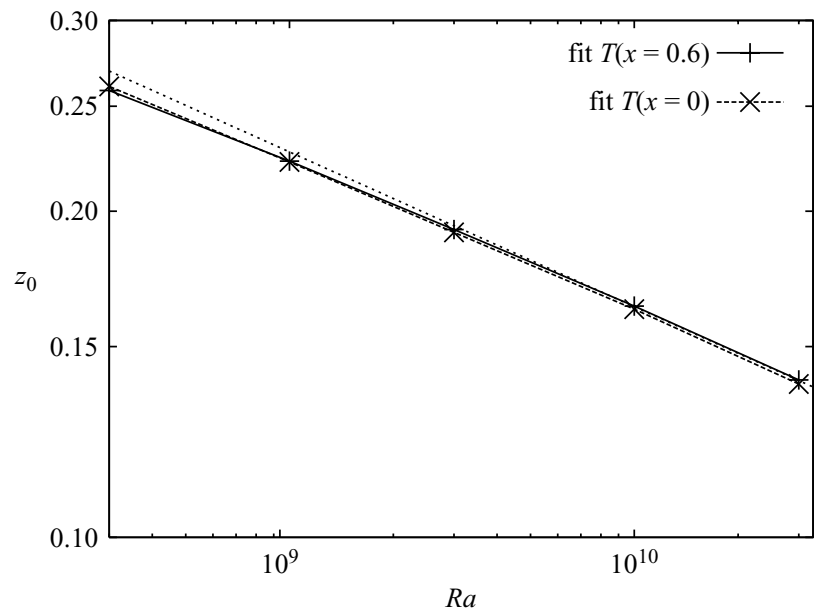

FiguRE 17. Offset $z_{0}$ for different Rayleigh numbers, found by fitting (4.6b) and (4.3b) to the numerical results at $x=0$ (dashed) and $x=0.6$ (solid), respectively. The suggested asymptote (dotted) is $z_{0}=4.38 R a^{-1 / 7}$.

approximate the asymptotic temperature $T_{b}$ at depth by the temperature in the bottom right corner.

To determine $z_{0}$, we fitted the power-law solutions for the wall and interior temperatures, $(4.6 b)$ with $c=6610$ and $(4.3 b)$ with $G(0)=-4980$, to the numerically determined temperature profiles at $x=0$ and at $x=0.6$, respectively. (The position $x=0.6$ was chosen since it lies roughly in the middle of the counter-rotating cells that can be seen superposed on the mean upwelling in figure $9(a)$, and the amplitude of the consequent oscillatory perturbation to the mean temperature profile is smaller there.) We fitted the profiles over the range $z_{0}<z<2 z_{0}$, with $z_{0}$ determined iteratively from an initial guess, say $z_{0}=0.2$. The converged values of $z_{0}$ from $x=0$ and $x=0.6$ are in excellent agreement (figure 17), suggesting that they are constrained well. The values are also described well by an estimated asymptotic behaviour

$$
z_{0}=4.38 R a^{-1 / 7} \text {. }
$$

From figure 18, we can see that approximating $z_{0}$ by (4.15) still produces a good fit between $(4.3 b),(4.6 b)$ and the numerically obtained temperature profiles, over nearly two decades of $R a$ and over most of the depth. Hence, for simplicity, we have used (4.15) for $z_{0}$ in all the figures that require it. Further comment on the asymptotic behaviour (4.15) is given below, in the discussion of matching at the end of this subsection.

Figure 19 shows excellent agreement between the similarity solution for the plume and the numerical simulations. In particular, the location and amplitudes of the minima and maxima in the temperature and velocity are accurately predicted. The strong downflow in the plume is driven by the very cold fluid that is falling down the left-hand wall from the corner. Some warmer fluid is dragged down with it, as seen in the weaker maximum in $G-c$ near $\eta=0.25$, and this warmer fluid bobs back up again as it detrains into the interior, as seen in the upwelling around $\eta=0.4$. The (decaying) oscillations of both $w$ and $\theta$ into the far field are consistent with the fact that $f_{\infty}$ satisfies the condition (4.14) for complex roots in (4.13). In the far field $w$ 

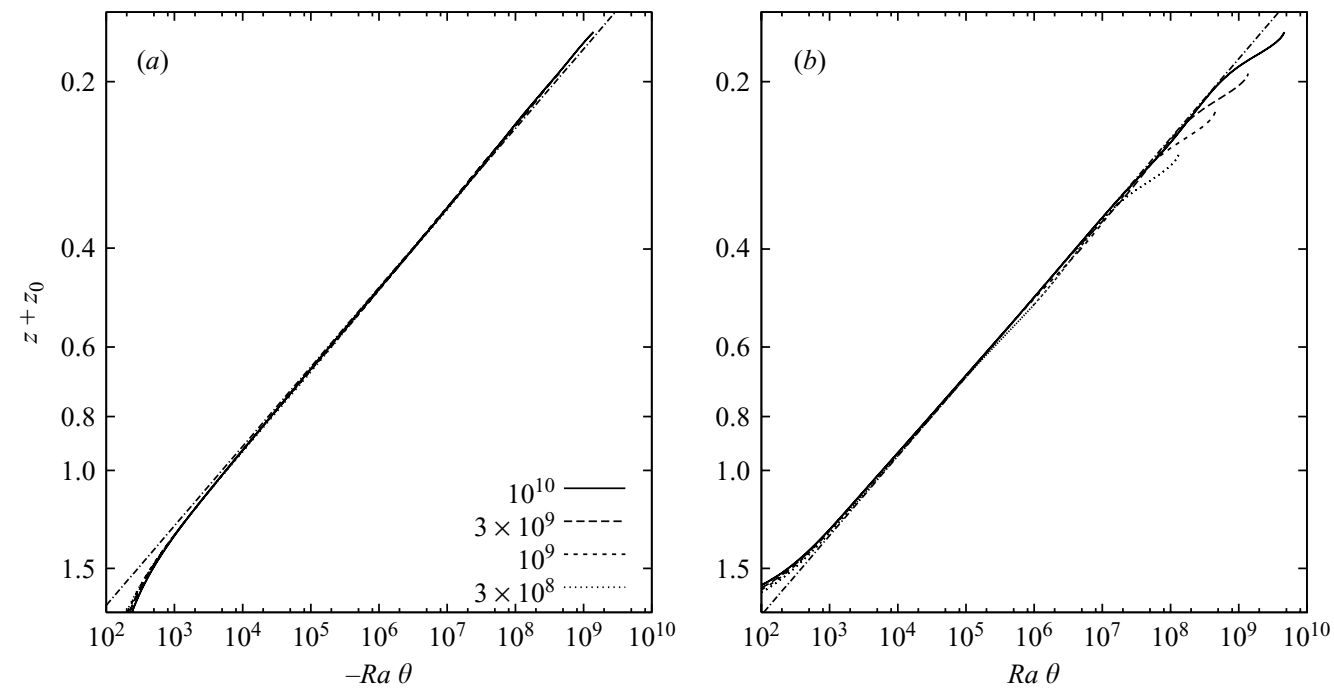

FIGURE 18. Vertical temperature profiles $(a) \theta(x=0, z)$ and $(b) \theta(x=0.6, z)$ for different Rayleigh numbers. The straight lines are $\operatorname{Ra} \theta=G(0) /\left(z+z_{0}\right)^{7}$ in $(a)$ and $R a \theta=c /\left(z+z_{0}\right)^{7}$ in $(b)$, where $G(0)=-4980$ and $c=6610$, as determined from the similarity solution for the sidewall plume.
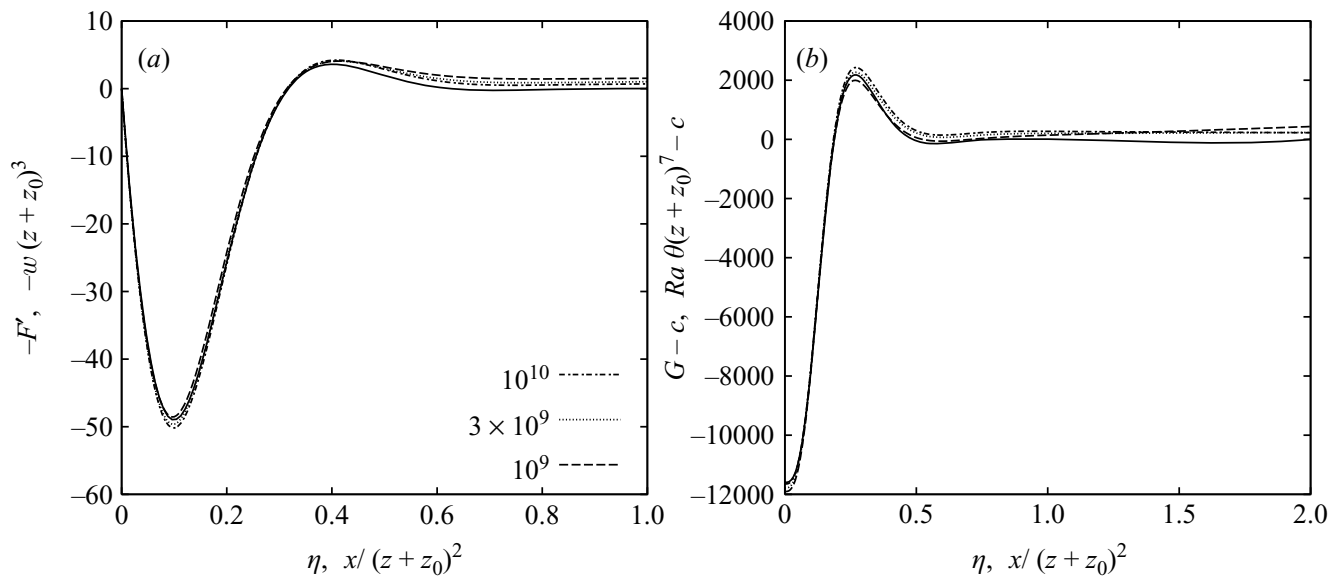

FIGURE 19. Similarity solutions (solid) for $(a)$ the plume velocity $-F^{\prime}$ and $(b)$ temperature difference $G-c$, where $c=6610$. There is good agreement with rescaled profiles from $z=z_{0}$ in full numerical solutions at different Rayleigh numbers.

and $\theta_{x}$ tend to zero in the similarity solution, and to interior values that are small relative to the variations in the plume in the full numerical solution.

Figure 20 shows that the numerically obtained plume width, which we define to be the location of the maximum downwards velocity, agrees well with the similarity solution for $z+z_{0} \lesssim 0.5$, but is a little narrower for $z+z_{0} \gtrsim 0.5$. The numerical solutions diverge from the similarity form when $z=O(1)$ because the plume width, $\left(z+z_{0}\right)^{2}$, is $O(1)$ at these depths, and hence is comparable to the width of the box. The lubrication approximation that was used in (4.2) thus breaks down, so the similarity solution is only expected to hold for $z \ll 1$. The slower growth of the plume width 


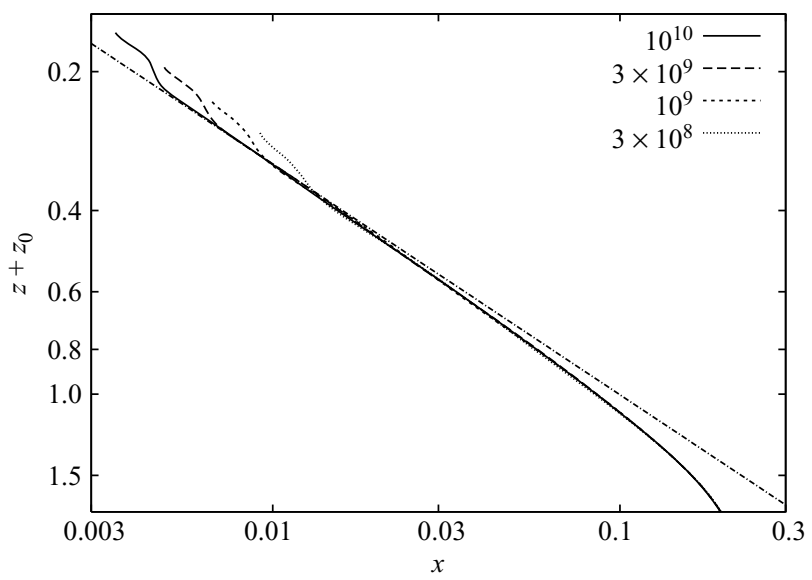

FIGURE 20. Plume width $x(z)$ defined as the position of the maximum downwards velocity.

The line $x=0.099\left(z+z_{0}\right)^{2}$ (dot-dashed) is given by the similarity solution (4.6).

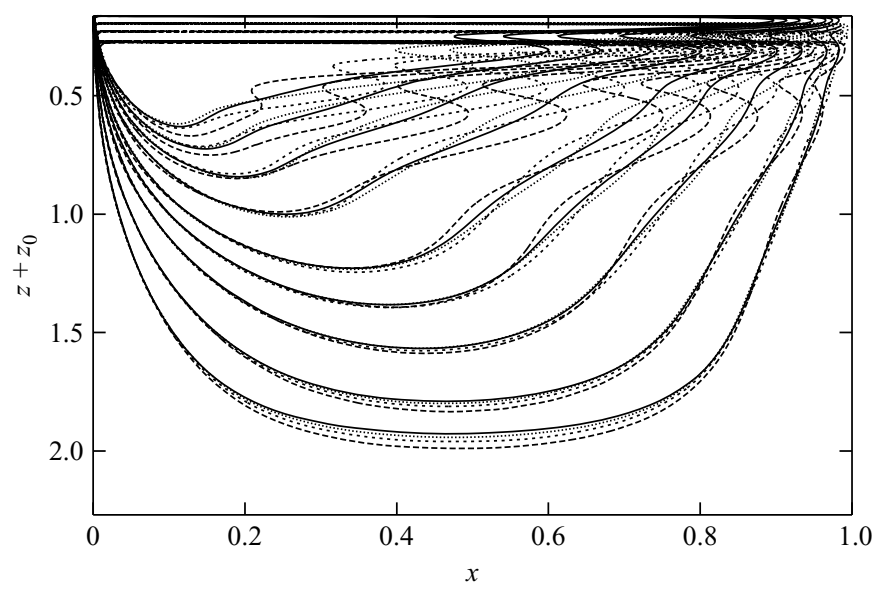

FIgURE 21. Interior flow. Streamlines $\psi=0.5,1,2,3,4,6,8,10,12$ at $R a=3 \times 10^{8}$ (long dashed), $10^{9}$ (dashed), $3 \times 10^{9}$ (dotted) and $10^{10}$ (solid).

when $z=O(1)$ helps to maintain the structure of a downwelling plume and stratified interior. At still greater depths, and if the bottom boundary is far enough away, the streamfunction and temperature difference are expected to decay exponentially with depth.

A second reason for the breakdown of the similarity solution when $z \sim 1$ is that at these depths, $\theta \sim T_{x} \sim T_{z} \sim 1 / R a$ and $\psi \sim 1$. Hence $T_{x}$ becomes comparable to $T_{z}$, so that the interior is no longer strongly stratified and the previous analysis does not apply.

Figure 21 shows the interior streamlines at different $R a$, with $\psi$ considered as a function of $x$ and $z+z_{0}$. Towards the top boundary, the influence of the counterrotating cells can be seen, but away from the top boundary the interior streamlines show good collapse. Small deviations also arise near the bottom boundary at $z=2$.

\section{CAMBridge JDURNALS}




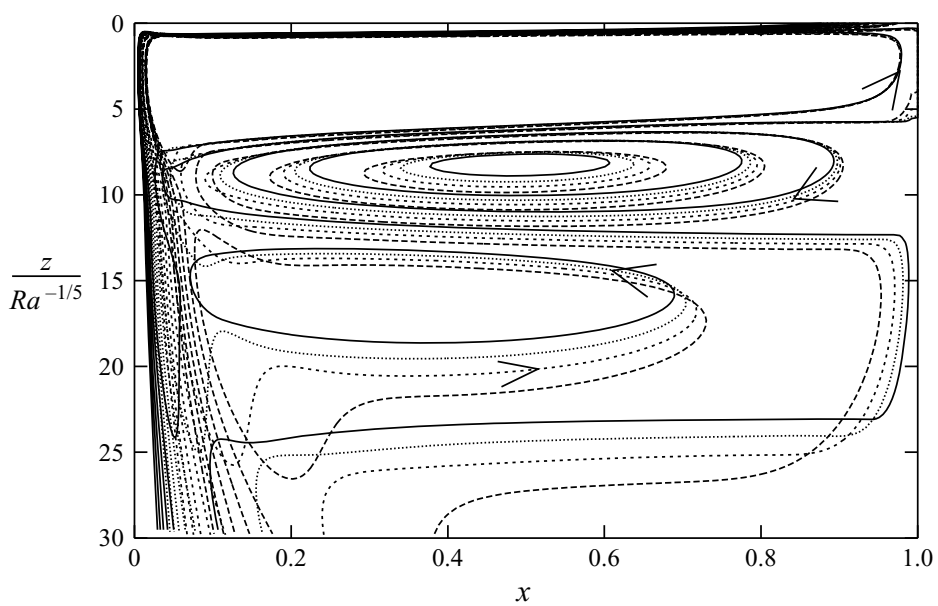

Figure 22. Cellular structure $\tilde{\psi}=\psi-8(1-x) /\left(z+z_{0}\right)$ for $R a=3 \times 10^{8}$ (long dashed), $10^{9}$ (dashed), $3 \times 10^{9}$ (dotted) and $10^{10}$ (solid), at contour levels $\tilde{\psi} / R a^{1 / 5}=-0.15,0,0.15,0.30$, 0.45 .

\section{Counter-rotating cellular structure}

From figure $9(a)$ we noted that the solution $-w=8 /\left(z+z_{0}\right)$ is a reasonable approximation over an intermediate range of heights $\left(0.25<z+z_{0}<0.8\right)$, but superposed on top of the leading-order solution is a smaller contribution from a stack of counter-rotating cells. Figure $9(a)$ showed that these cells lie beneath the surface boundary layer and intrusion, and decay with depth. The structure of the cells is most readily revealed by plotting the perturbation

$$
\tilde{\psi}=\psi-\frac{8(1-x)}{z+z_{0}}
$$

to the uniform upwelling (4.3), as shown in figure 22. The scales for $\tilde{\psi}$ and the vertical coordinate have been chosen to give agreement with the $\mathrm{Ra}^{1 / 5}$ scalings in the top cell, which is the boundary layer and intrusion. Beneath the top cell, we can see at least two weaker counter-rotating cells, which decrease in strength with depth.

Some insight into why the cellular structure exists can be gained by considering small perturbations $\tilde{T}$ and $\tilde{\psi}$ to the background stratification $T_{0}(z)$ and upwelling (4.3). The linearization of (2.2) about the background stratification is

$$
\tilde{\psi}_{x} T_{0 z}=\tilde{T}_{z z},
$$

which can be combined with (2.1) to yield

$$
\tilde{\psi}_{z z z z z z}=\operatorname{Ra} T_{0 z} \tilde{\psi}_{x x} .
$$

Since the background stratification $T_{0 z}$ only depends on the depth $z$, this equation has separable solutions of the form $\tilde{\psi}=\sin (n \pi x) f_{n}(z)$, where $f_{n}$ is decaying and oscillatory in $z$. For $z \ll 1$, the dependence of $R a T_{0 z}$ on $z+z_{0}$ in (4.3) implies that the vertical scale of the oscillations is small, in qualitative agreement with figure 22 . The counter-rotating cells are driven by the shear stress from the intrusion, and then decay with depth. 

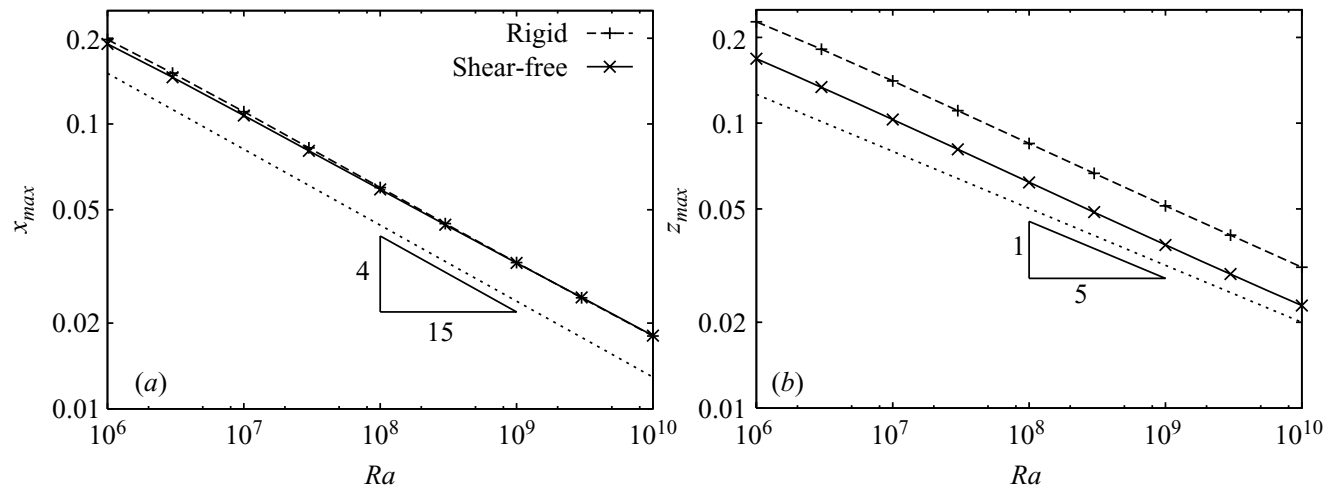

FIGURE 23. The position of the streamfunction maximum $\left(x_{\max }, z_{\max }\right)$ against $R a$ for $A=1$ in the boundary-layer regime. The power laws (dotted) are those predicted by scaling arguments.

\section{Corner region}

We denote the position of the streamfunction maximum by $\left(x_{\max }, z_{\max }\right)$. From figure 23, we can see that both $x_{\max }$ and $z_{\max }$ follow approximate power-law scalings with $R a$, with $x_{\max }$ decreasing more rapidly with $R a$ than $z_{\max }$. This thinning of the flow as it comes round the top left corner is driven by the negative buoyancy of sinking cold fluid. From (2.1), and an assumption that the temperature differences are $O(1)$ as in the boundary layer, the vorticity balance in the corner gives

$$
\frac{\psi}{x^{4}} \sim \frac{R a}{x} .
$$

Since the volume flux arriving from the boundary layer is $\psi \sim R a^{1 / 5}$, we predict that $x_{\max } \sim R a^{-4 / 15}$, in reasonable agreement with figure $23(a)$. Figure $23(b)$ shows that the expected scaling $z_{\text {max }} \sim R a^{-1 / 5}$ is also asymptotically reasonable.

Comparison of $\psi_{z} T_{x}$ to $T_{x x}$ with the above scalings shows that thermal advection dominates diffusion in the corner region, except near the positions of the maximum in the streamfunction and of the stagnation point in the corner, at both of which the velocity is zero. A small diffusive subregion near the corner is required to match to the insulating boundary condition on the sidewall. If we assume that the flow in the corner can be approximated by a rigid-wall stagnation-point flow $\psi \sim R a^{14 / 15} x^{2} z$, where the dependence on $R a$ is determined by matching to $\psi_{\max } \sim R a^{1 / 5}$ at $\left(x_{\max }\right.$, $z_{\max }$ ), then a balance $u T_{x} \sim T_{x x}$ between advection towards and diffusion away from the wall shows that the diffusive boundary layer on the left-hand side has thickness $x \sim R a^{-14 / 45}$. (For a shear-free side a stagnation-point flow $\psi \sim R a^{2 / 3} x z$ leads to a thickness $x \sim R a^{-1 / 3}$.) This thickness is numerically indistinguishable from the width $x_{\text {max }} \sim R a^{-4 / 15}$ of the corner region.

\section{Matching}

We have already noted that the analytical similarity solutions obtained for the plume and interior are only valid for an intermediate range of heights $R a^{-\alpha} \ll z \ll 1$. The condition $z \ll 1$ comes from requiring the plume width to be small compared to the width of the box. The condition $z \gg R a^{-\alpha}$ comes from the need to match the plume back to the corner region and the stratified interior up to the top boundary layer. Now the similarity forms (4.6b) and $(4.3 b)$ for the plume and interior must break down for $z+z_{0} \ll R a^{-1 / 7}$ since otherwise $\theta \gg 1$ and this would be inconsistent with the 


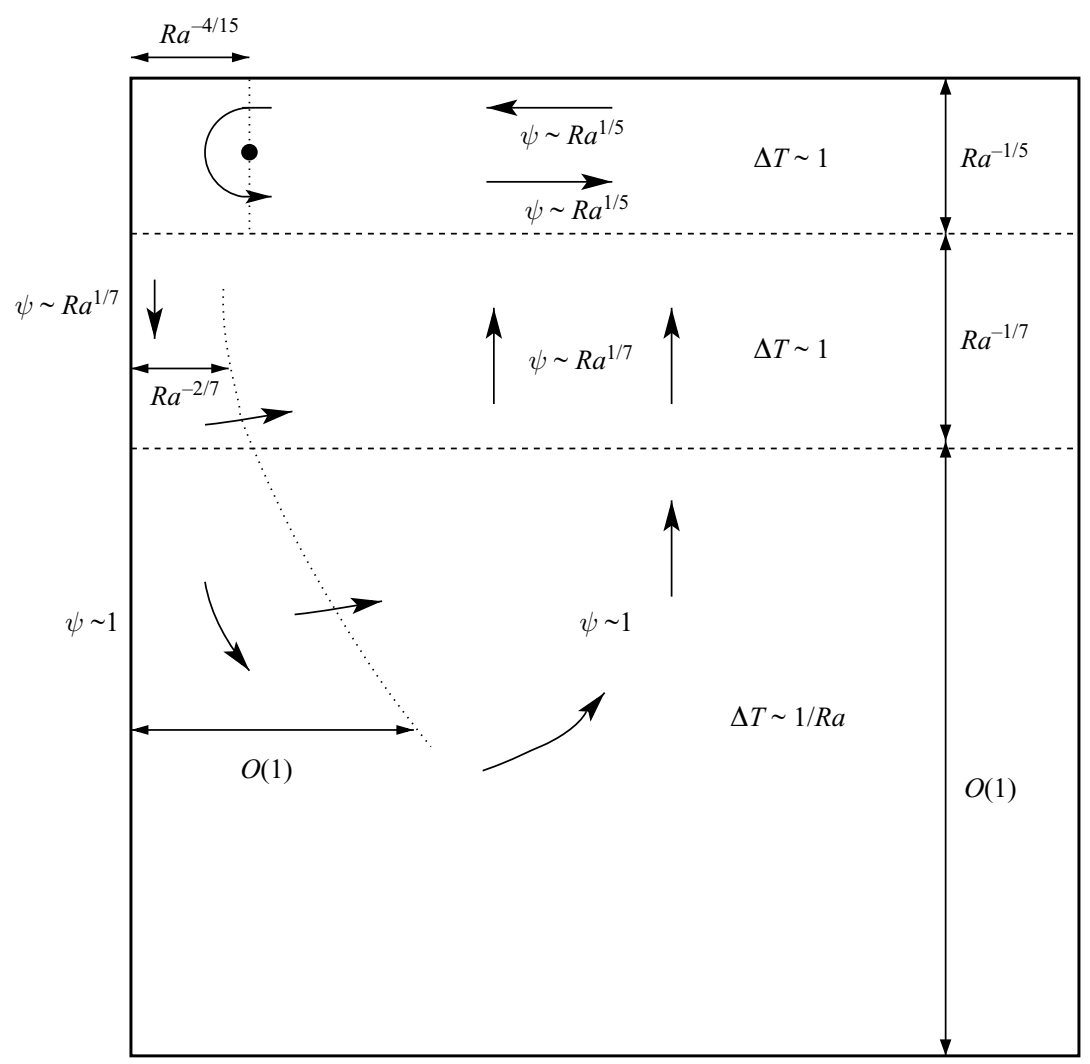

FIGURE 24. Schematic of the different regions of the flow in the large- $R a$ regime, with the corresponding scalings of the temperature variations and streamfunction. The similarity solution for the plume and the solutions of a strongly stratified interior with a uniform upwelling are valid for $R a^{-\alpha} \ll z \ll 1$, where $\alpha$ is assumed to be $1 / 7$.

range of applied surface temperature. Hence if we make the natural assumption that the vertical offset $z_{0}$ is comparable to the scale of the matching region, i.e. $z_{0} \sim R a^{-\alpha}$, then it follows that $\alpha \leqslant \frac{1}{7}$.

The good empirical fit (4.15) to the numerical data for $z_{0}$ in figure 17 is consistent with $\alpha=\frac{1}{7}$ as used in (4.15) and, for simplicity of exposition below, we shall assume this to be the correct value. We note that the data could also be consistent with a slightly smaller value of $\alpha$ - the last three data points in figure 17 give $\alpha=0.139 \pm 0.004$, and $z_{0}$ is itself a fitted quantity and subject to a small uncertainty.

The scalings of the various regions of the flow are summarized in figure 24. Since the thickness of the top boundary layer $\sim R a^{-1 / 5}$ but the analytical solutions for the interior are only valid at a minimum depth $\sim R a^{-1 / 7}$ (and possibly slightly larger), the present analysis leaves a gap in heights between $R a^{-1 / 5}$ and $R a^{-1 / 7}$. Also, the $R a^{-4 / 15}$ width of the corner region does not match directly onto the minimum width $\sim R a^{-2 / 7}$ of the similarity solution for the plume at depth $\sim R a^{-1 / 7}$.

A complete theoretical analysis should describe how the $\mathrm{Ra}^{-4 / 15}$ outflow from the corner region, together with its $R a^{-4 / 45}$ diffusive sublayer, separates into the intrusion and the plume, and how the counter-rotating cellular structure decays from the strong top boundary layer and intrusion into a small perturbation on a stratified interior. We were unable to find a convincing matching argument to bridge the small asymptotic 

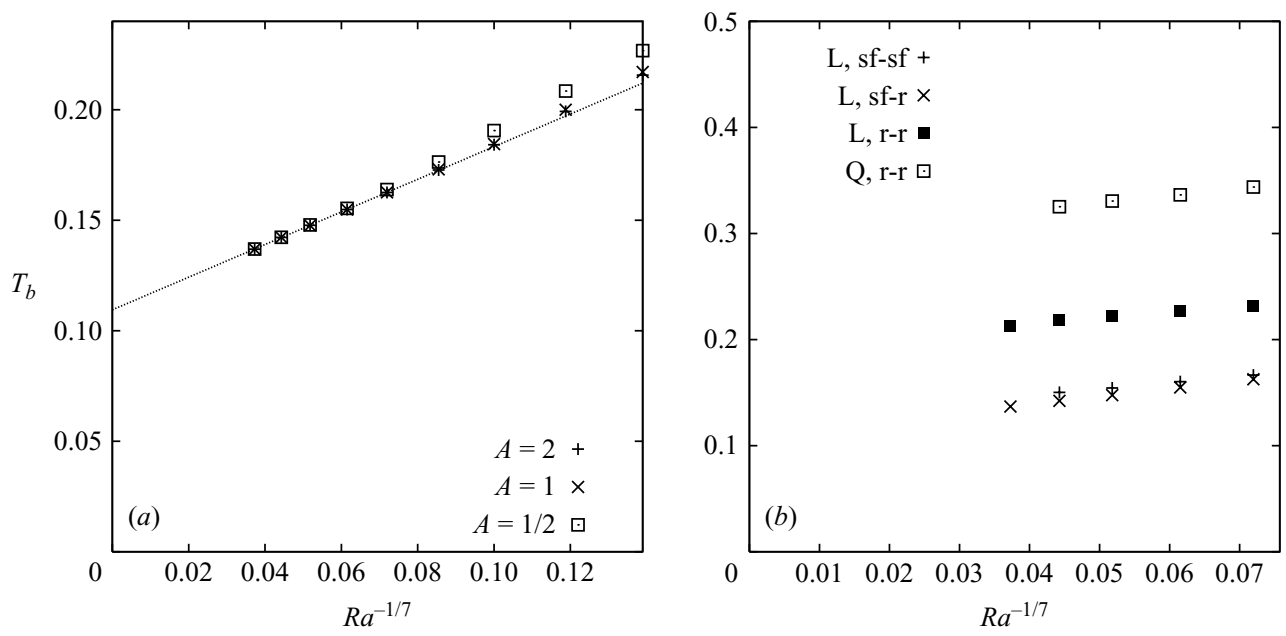

FiguRE 25. Temperature $T_{b}$ in the bottom right corner, for $(a)$ different aspect ratios with a shear-free top, rigid sides and bottom, and a linear temperature profile $T=x$ on the top surface; and $(b)$ different boundary conditions with $A=1$, where e.g. sf-r denotes shear-free top and rigid sides and bottom, $\mathrm{L}$ denotes the linear profile $T=x$ and $\mathrm{Q}$ the quadratic profile $T=x(2-x)$ on the top surface.

gap between the near-surface and deep structures. Various attempts gave plausible scalings for the intermediate region, but it was impossible to distinguish between them numerically. Even for $R a=10^{10}, R a^{1 / 5}$ and $R a^{1 / 7}$ differ by only a factor 4 and thus the intermediate region cannot be clearly separated. However, our numerical simulations provide good evidence that the present analysis, though incomplete, does give the scalings and solutions for the main regions of the flow.

\subsection{Interior temperature and effect of aspect ratio}

The solutions for the interior temperature differences derived in $\S 4.3$ included the reference temperature $T_{b}$, the asymptotic temperature at depth, which we approximated by the bottom-right temperature of the numerical simulations. Since the interior temperature variations are small below the top boundary, decaying rapidly as $\left(z+z_{0}\right)^{-7}, T_{b}$ is a good approximation for the temperature of the nearly isothermal interior.

Figure 25(a) shows $T_{b}$ as a function of $R a^{-1 / 7}$ for a number of different aspect ratios. For all values of $A$, the interior temperature decreases as $R a$ increases, which reflects the increased flow strength in the boundary layer. The consequent increase in the strength of upward entrainment leads to less diffusion of the warmer surface temperature to the interior. The data for $A=1$ and $A=2$ are indistinguishable, which gives numerical evidence that $T_{b}$ becomes independent of $A$ as $A \rightarrow \infty$, and is determined only by the surface structure near the top boundary. At smaller values of $R a$, say $10^{7}$, the proximity of the bottom boundary to the top results in an increased bottom-right temperature for $A=1 / 2$.

Figure 25(b) shows $T_{b}$ as a function of $R a^{-1 / 7}$ for a number of different boundary conditions on the top and on the sides and bottom. Changes to the top boundary conditions, whether from a linear to quadratic temperature profile or from shear-free to rigid, have a much greater effect on the interior temperature than either a change from rigid to shear-free conditions on the sides and bottom or the variation over two decades of Rayleigh number. The significant increase in the interior temperature 

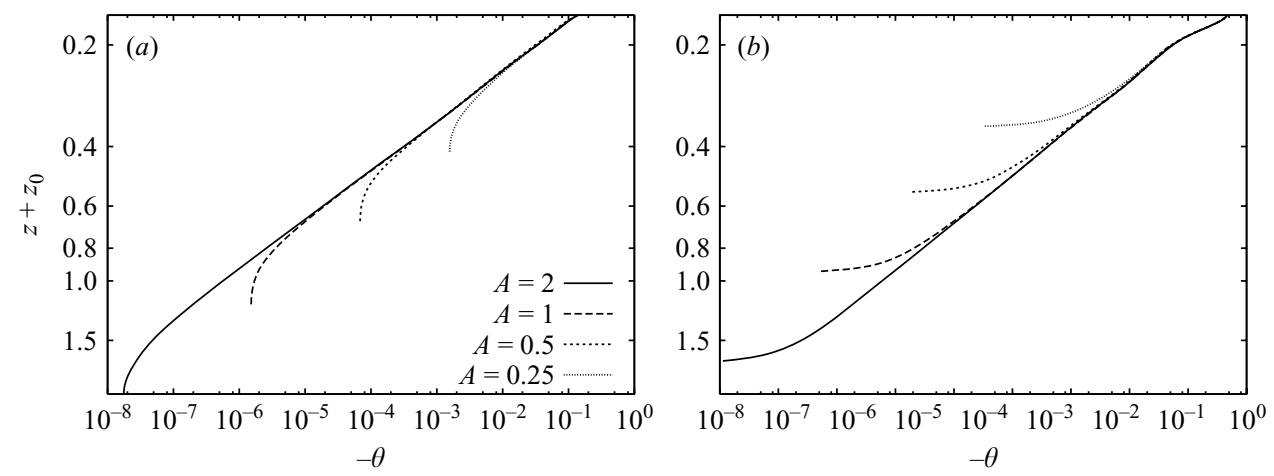

Figure 26. Temperature variations relative to the temperature $T_{b}(A)$ in the bottom right corner at $(a) x=0$ and $(b) x=0.6$, for different aspect ratios and $R a=10^{10}$.

for a rigid top or for the quadratic profile $T=x(2-x)$ reflects the reduction in the strength in the right half of the surface boundary layer. The consequent reduction in the strength of upward entrainment allows the warmer surface temperature to diffuse to great depths. The variation with boundary condition suggests that the interior temperature is primarily determined by the balance between upward entrainment into the surface boundary layer and downward diffusion, though the rate of entrainment is also influenced by the stratification established by the weak circulation through the plume to the interior.

The choice of $R a^{-1 / 7}$ as an axis in figure 25 is motivated partly by convenience and partly by the apparent form of the vertical offset $z_{0}$. It gives a good straight-line asymptote for the data with $A=2$ ( or $A=1$ ) in figure 25( $a$ ), which can be extrapolated to give $T_{b} \rightarrow 0.11$ as $R a \rightarrow \infty$. The actual asymptotic form of the variation of $T_{b}$ with $R a$ is unknown, and would need to be determined by a full matching analysis of the near-surface and deep structures.

The temperature at $x=0$ and $x=0.6$ relative to the bottom-right corner is plotted in figure 26 for different aspect ratios. The temperature profiles for $A \leqslant 1$ follow the temperature profile for $A=2$ up to $z \lesssim A / 2$, and then at $z \approx A / 2$ the bottom boundary begins to have an influence and the curves peel off. Similarly, as shown in figure 27, the streamlines for different aspect ratios follow those of the tall box $A=2$ for $z \lesssim A / 2$ and then the streamlines peel off.

\section{Discussion}

We have analysed very viscous horizontal convection for a wide range of aspect ratio and Rayleigh number. The transition between the linear or intermediate regimes and the boundary-layer regime is governed by $A^{5} R a$ if $A \ll 1$ and by $R a$ if $A \geqslant 1$. For steady states at large Rayleigh number, we have provided a description of the several regions of the flow, and explained the scalings for the widths of the boundary layers, the temperature variations and the streamfunction.

At large Rayleigh number, the independence of the scalings of whether the top boundary is rigid or shear-free is at first surprising, as is the weakness of the flow at depth, but both can be understood from the global structure of the flow. This structure is summarized in figure 10, with the corresponding scalings in the different regions detailed in figure 24 . The sinking plume on the left-hand wall decays rapidly with depth because of the strong stratification of the interior, and most of the return 


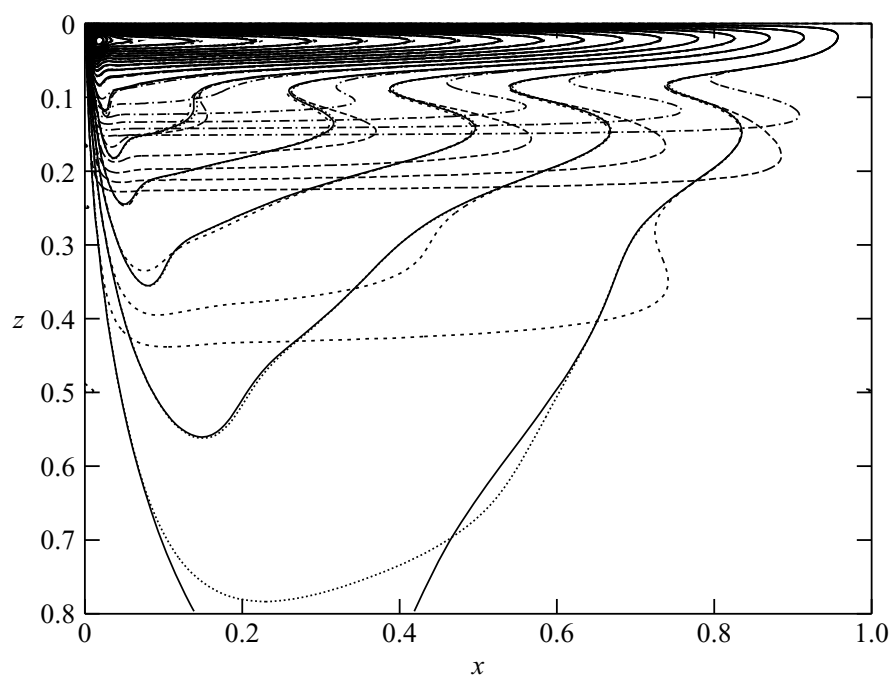

FIGURE 27. Streamlines in increments of 5 at $R a=10^{10}$, and different aspect ratios $A=1 / 6$ (dot-dash), 1/4 (long dashed), 1/2 (dashed), 1 (dotted) and 2 (solid).

flow comes back in a shallow intrusion. The intrusion therefore gives strong shear in the boundary layer even for a shear-free surface condition and, as a consequence, the scalings are independent of whether the top boundary is rigid or shear-free. The dominance of the return flow by a shallow intrusion is supported by the experimental observations of Wang \& Huang (2005), who found 'partial penetration' of the return flow into the interior, with most of the flow confined to near the heated surface.

The sidewall plume and interior form a viscous 'filling box'. In $\S 4.3$, we found a similarity solution for the filling box that is valid for $R a^{-\alpha} \ll z \ll 1$, and gave numerical evidence that $\alpha=1 / 7$. The similarity solution gives $R a T \sim\left(z+z_{0}\right)^{-7}$ in both the plume and interior, and $w \sim\left(z+z_{0}\right)^{-3}$ in the plume and $\left(z+z_{0}\right)^{-1}$ in the interior, where $z_{0} \sim R a^{-1 / 7}$. This solution does not depend on the details of the flow at the top, and may be more generally applicable (e.g. to top boundary conditions of imposed heat flux). We have not been able to match the interior solution directly onto the horizontal boundary layer or corner flow. A complete theoretical analysis should describe how the flow exiting the corner separates into the intrusion and plume, and also the underlying cellular structure and the matching between the horizontal boundary layer and interior.

Although we have primarily presented results with rigid side and bottom boundaries, computations with all the boundaries shear-free show very similar results. The main modification that needs to be made in the theoretical analysis is the boundary condition (4.11) for the plume. Hence the structure and scalings we have derived (see figure 24) are independent of the mechanical conditions on the boundaries. Also, the exact geometry is unlikely to be important, provided the heated surface is horizontal. For example, sloping sidewalls will give a similar structure and the same scalings.

The present asymptotic analysis is in a similar spirit to that of a study of horizontal convection in a porous medium by Daniels \& Punpocha (2005). These authors show that in a porous medium the horizontal boundary layer has width $\delta \sim R a^{-1 / 3}$ instead of $R a^{-1 / 5}$ here. As in the present work, they find power-law decays in $z$, with $\psi \sim 1 / z$, and $R a \theta \sim 1 / z^{3}$ instead of $1 / z^{7}$. A significant difference is that in a porous medium 
the interior is not strongly stratified and all the terms in the heat equation (2.2) need to be retained. Hence the upwelling velocity and temperature are not uniform across the width of the box.

For modelling of viscous horizontal convection in applications, several other effects may need to be taken into account. For results with a highly temperature-dependent viscosity (not presented here), we find that the solutions for the interior are not significantly affected, since the temperature variations there are small, but the horizontal boundary layer is quantitatively modified. Future work could consider heat losses through the walls and three-dimensional geometry.

Despite the idealizations in the present work, it is interesting to consider the relevance of the ideas to glass furnaces, which provided the original motivation for the problem. The glass industry uses shallow tanks $(A \ll 1)$ and, to aid convective transfer of heat underneath the entire length of floating cold raw materials, it is thus helpful to operate in the boundary-layer regime. Hence we require that $A^{5} R a \gtrsim 2 \times 10^{3}$ (figure 5). However, if the value of $A^{5} R a$ is too large then a separation of scales will exist between the top boundary layer and a stratified interior, leading to poor mixing in the slow-moving interior and a risk of heterogeneities in the glass produced. A reasonable requirement is to use a small enough value of $A^{5} R a$ that the depth of the tank is just smaller than the depth of the intrusion, which from figure 16 leads to $A / R a^{-1 / 5} \lesssim 10$, or $A^{5} R a \lesssim 10^{5}$. In glass furnaces, $A^{5} R a \approx 10^{4}$ (Kraus \& Loch 2002), which is consistent with $2 \times 10^{3} \lesssim A^{5} R a \lesssim 10^{5}$.

For the large- and infinite-Prandtl-number calculations reported here, the final steady state for a shear-free top was found to be independent of the initial conditions, even if the initial conditions are as disparate as those of figures 11 and 12, which suggests that this final steady state is stable. Without fluid inertia the only plausible instability would be in the upper left part of the top boundary layer, where cold fluid near the top surface overlies hotter fluid. For the two-dimensional calculations reported here, the velocity in the boundary layer with a shear-free top was apparently sufficiently large to avoid instability. In three dimensions, however, the unstable stratification may lead to overturning rolls with axes aligned along the flow direction and this instability may occur at only moderately large Rayleigh number. By contrast with the shear-free case, finite-Prandtl-number simulations for a rigid top did not evolve to a steady state for $R a \geqslant 3 \times 10^{8}$, even at $\operatorname{Pr}=10^{5}$, owing to instability in the upper left part of the boundary layer. Presumably, this is because the no-slip condition allows time for local convective overturning in the boundary layer. Unsteady and three-dimensional solutions remain topics for future investigation.

At finite Prandtl number, the effects of shear and inertia will almost certainly lead to further instabilities at sufficiently large Rayleigh number. The vorticity equation (2.1) becomes

$$
\frac{1}{\operatorname{Pr}} \frac{\partial\left(\nabla^{2} \psi, \psi\right)}{\partial(x, z)}+\nabla^{4} \psi=R a \frac{\partial T}{\partial x},
$$

from which we can define a local Reynolds number as the ratio of the typical magnitude of the two terms on the left-hand side. The largest local Reynolds number in our analysis comes from the downflow in the corner region, and scales as $\psi_{x} x^{2} / z \operatorname{Pr} \sim R a^{2 / 15} / P r$. In contrast, the local Reynolds numbers in both the horizontal boundary layer $(\sim \psi \delta)$ and the side plume are only $1 / P r$. Hence, in the boundary-layer regime inertial instability might be expected first in the corner region.

Finite-Prandtl-number effects are particularly important for the Rayleigh numbers of $10^{11}-10^{13}$ considered by Mullarney et al. (2004) with $\operatorname{Pr} \simeq 8$. Indeed, they observe 
a much stronger, unsteady plume that entrains fluid, and reaches and spreads along the opposite wall of the tank. They also report the presence of a convective mixed layer in the horizontal boundary layer, weak interior mixing, and unsteady and apparently turbulent flow. Hughes et al. (2007) present a model for the results of Mullarney et al. (2004), and have shown that the $R a^{1 / 5}$ scalings of the top boundary layer still hold (or $R a^{1 / 6}$ scalings for the case of imposed heat flux). However, the intrusion is insignificant, and the return flow is characterized by the turbulent plume, which is coupled to a filling-box model of the interior. Near the heated surface the plume is driven by buoyancy and modified by entrainment, whereas away from the surface it becomes a momentum jet with entrainment. The approximation (4.1) for the interior balance still applies. However, the interior is only weakly stratified because the plume/jet is driven to depth and coupled to the interior by turbulent entrainment. The entrainment also leads to the volume flux in the plume increasing with depth like $z^{1 / 2}$. These results contrast with the strong stratification of the interior, the detrainment of the plume and the $z^{-1}$ decay of the volume flux in the infinitePrandtl-number case.

From the previous and current results, it appears that there is a trade-off between Prandtl and Rayleigh number: at large $R a$ we expect that the intrusion dominates the return flow for sufficiently large $\operatorname{Pr}$ (Wang \& Huang 2005; present results), but at fixed $\mathrm{Pr}$ a turbulent plume dominates the return flow for sufficiently large $R a$ (Mullarney et al. 2004; Hughes et al. 2007).

To conclude, a physical understanding of infinite-Prandtl-number horizontal convection has been achieved, and the main scaling relationships derived. In particular, we have identified that at large Rayleigh numbers the return flow is dominated by a warm shallow intrusion above a cool strongly stratified interior.

We thank Neil Ribe, Grae Worster, and three anonymous referees for helpful comments on an earlier version of the manuscript. S. Chiu-Webster was supported by an EPSRC research studentship.

\section{REFERENCES}

Blankenbach, B., Busse, F., Christensen, U., Cserepes, L., Gunkel, D., Hansen, U., Harder, H., Jarvis, G., Koch, M., Marquart, G., Moore, D., Olson, P., Schmeling, H. \& Schnaubelt, T. 1989 A benchmark comparison for mantle convection codes. Geophys. J. Int. 98, 23-38.

Daniels, P. G. \& PunPoCha, M. 2004 Cavity flow in a porous medium driven by differential heating. Intl J. Heat Mass Transfer 47, 3017-3030.

Daniels, P. G. \& PunPocha, M. 2005 On the boundary-layer structure of cavity flow in a porous medium driven by differential heating. J. Fluid Mech. 532, 321-344.

Davis, R. H., Schonberg, J. A. \& Rallison, J. M. 1989 The lubrication force between two viscous drops. Phys. Fluids A 1, 77-81.

Doedel, E. J., Champneys, A. R., Fairgrieve, T. F., Kuznetsov, Y. A., Sandstede, B. \& Wang, X.-J. 1997 AUTO97: Continuation and bifurcation software for ordinary differential equations. Available by FTP from ftp.cs.concordia.ca in directory pub/doedel/auto.

Gramberg, H. J. J., Howell, P. D. \& OCKendon, J. R. 2007 Convection by a horizontal thermal gradient. J. Fluid Mech. 586, 41-57.

Huang, R. X. 1999 Mixing and energetics of the oceanic thermohaline circulation. J. Phys. Oceanogr. 29, 727-746.

Hughes, G. O. \& GRIfFiths, R. W. 2006 A simple convective model of the global overturning circulation, including effects of entrainment into sinking regions. Ocean Modelling 12, 46-79.

Hughes, G. O., Griffiths, R. W., Mullarney, J. C. \& Peterson, W. H. 2007 A theoretical model for horizontal convection at high Rayleigh number. J. Fluid Mech. 581, 251-276. 
Huppert, H. E. 1982 The propagation of two-dimensional and axisymmetric viscous gravity currents over a rigid horizontal surface. J. Fluid Mech. 121, 43-58.

Killworth, P. D. \& Manins, P. C. 1980 A model of confined thermal convection driven by non-uniform heating from below. J. Fluid Mech. 98, 587-607.

Kraus, D. \& LoCh, H. (Ed.) 2002 Mathematical Simulation in Glass Technology. Springer.

Lister, J. R. \& KerR, R. C. 1989 The propagation of two-dimensional and axisymmetric viscous gravity currents at a fluid interface. J. Fluid Mech. 203, 215-249.

McKenzie, D. P., Roberts, J. M. \& Weiss, N. O. 1974 Convection in the earth's mantle: towards a numerical simulation. J. Fluid Mech. 62, 465-538.

Mullarney, J. C., Griffiths, R. W. \& Hughes, G. O. 2004 Convection driven by differential heating at a horizontal boundary. J. Fluid Mech. 516, 181-209.

Paparella, F. \& Young, W. R. 2002 Horizontal convection is non-turbulent. J. Fluid Mech. 466, 205-214.

Roberts, G. O. 1977 Fast viscous convection. Geophys. Astrophys. Fluid Dyn. 8, 197-233.

RossBy, T. 1965 On thermal convection driven by non-uniform heating from below; an experimental study. Deep-Sea Res. 12, 9-16.

Rossby, T. 1998 Numerical experiments with a fluid heated non-uniformly from below. Tellus 50A, $242-257$.

Siggers, J. H., Kerswell, R. R. \& Balmforth, N. J. 2004 Bounds on horizontal convection. J. Fluid Mech. 517, 55-70.

WANG, W. \& HuANG, R. X. 2005 An experimental study on thermal circulation driven by horizontal differential heating. J. Fluid Mech. 540, 49-73.

Worster, M. G. \& Leitch, A. M. 1985 Laminar free convection in confined regions. J. Fluid Mech. 156, 301-319.

Wunsch, C. \& Ferrari, R. 2004 Vertical mixing, energy, and the general circulation of the oceans. Annu. Rev. Fluid Mech. 36, 281-314. 